\title{
Intuitionen als Gründe? Das Problem der intuitiven Rechtfertigung
}

\subsection{Zur Plausibilität der Rechtfertigung durch Intuition}

Ist es statthaft auf die Frage »Woher weißt du, dass du existierst? « zu antworten: »Weil ich die entsprechende Intuition habe«? Bei dieser Frage geht es um die epistemische Rechtfertigung durch Intuition. ${ }^{181}$ Ist Intuition dazu geeignet, sich der Wahrheit anzunähern und Irrtum zu vermeiden? ${ }^{182}$ Wer dies verneint, ohne im Sinne eines starken Skeptizismus die Möglichkeit epistemischer Rechtfertigung überhaupt negieren zu wollen, nimmt die Position ein, wonach epistemische Rechtfertigung nur auf diskursivem Weg möglich ist. In Bezug auf das Cartesische Beispiel würde somit die Rechtfertigung den Prämissen eines deduktiven Arguments entsprechen: »Ich bin ein denkendes Wesen, alle denkenden Wesen existieren, also existiere ich.« Falls epistemische Rechtfertigung nur auf diskursivem Weg erfolgen kann, hat die Intuition in epistemologischer Hinsicht keine oder nur eine untergeordnete Bedeutung. Der Intuitionsbegriff würde lediglich einen Denkstil oder eine Technik bezeichnen, etwa eine schnelle Art des Schlussfolgerns, und keine besondere Erkenntnisart. ${ }^{183}$ Dies steht jedoch nicht in Einklang mit dem verbreiteten Verständnis, wonach Intuitionen rechtfertigende Kraft haben. So sind wir in vielen Beispielen dazu bereit, Intuitionen als Gründe anzuerkennen.

Hier ein Beispiel für Rechtfertigung durch Intuition: Athletinnen und Athleten haben oft sehr gute Intuitionen bezüglich der Ausübung ihres Sports. Man denke beispielsweise an eine Golfspielerin, die intuitiv das jeweils beste Eisen für den nächsten Schlag wählt. >Intuitiv< kann hier zweierlei bedeuten:

181 Mit >Rechtfertigung< ist auch im Folgenden stets epistemische Rechtfertigung gemeint. Im Kontrast dazu stehen andere Arten der Rechtfertigung, die sich nicht auf das Erlangen von Wissen beziehen. Beispielsweise kann die Überzeugung eines Schwerkranken, dass bald ein Heilmittel gegen seine Krankheit gefunden werden wird, insofern gerechtfertigt sein, als sie ihm Hoffnung und Kraft gibt, weiterzuleben. Dies ist aber von einer epistemischen Rechtfertigung dieser Überzeugung zu unterscheiden (vgl. Fumerton 2002, S. 205; Kvanvig 2011, S. 26).

182 Vgl. Kvanvig (2011, S. 25).

183 Vgl. Gabriel (2015a, S. 64). 
erstens, dass die Golfspielerin aufgrund ihrer Intuition direkt handelt, indem sie das Eisen aus der Schlägertasche nimmt und abschlägt; zweitens, dass die Golfspielerin aufgrund ihrer Intuition zu einer Überzeugung kommt, die sich auf die Wahl des Eisens bezieht, etwa: »Wenn ich hier einen erfolgreichen Schlag erzielen will, muss ich Eisen 7 wählen «. Nehmen wir vorübergehend an, hier wäre keine Gründe gebende Intuition im Spiel, dann blieben drei Möglichkeiten: Erstens könnte man der Golfspielerin eine Überzeugung zuschreiben, die einen Grund für die Zielüberzeugung bzw. Handlung darstellt (etwa: »Wenn die Windbedingungen so und die Bodenbeschaffenheit so sind, dann ist es am besten Eisen $7 \mathrm{zu}$ nehmen «). Zweitens könnte man die Auffassung vertreten, dass weder eine Intuition noch eine Überzeugung in Bezug auf die Wahl des Eisens im Spiel sind. Drittens könnte man der Golfspielerin zwar eine Intuition (statt einer Überzeugung) bezüglich der Wahl des Eisens zuschreiben, jedoch verneinen, dass diese Intuition ein Grund für die Zielüberzeugung oder Handlung ist.

Die ersten beiden der drei genannten Möglichkeiten sind unplausibel: Der erste Fall ist zwar denkbar, aber kaum jemand dürfte dazu bereit sein, in allen derartigen Fällen den Subjekten Überzeugungen zuzuschreiben. Dies würde bedeuten, das Phänomen der Intuition, wie es in den vorigen Kapiteln beschrieben wurde (man denke an die Merkmale >Unmittelbarkeit< und $>$ Gewissheit<), zu negieren. Ähnliches gilt für den zweiten Fall: Dieser könnte auf eine kausale Erklärung hinauslaufen, die eine direkte Verbindung von Gehalten der Erfahrung (die Golfspielerin fühlt die Windrichtung, sieht die Bodenbeschaffenheit) zur entsprechenden Handlung bzw. Überzeugung erstellt. Würden aber alle dem geschilderten Beispiel ähnlichen Fälle auf diese Weise erklärt, könnten wir sie beispielsweise nicht von Fällen abgrenzen, in denen Subjekte fremdgesteuert sind; etwa, wenn wir die Golfspielerin hypnotisiert hätten und sie aufgrund der Hypnose zu Eisen 7 greift. Schon aufgrund solcher Abgrenzungsprobleme ist die zweite Möglichkeit zurückzuweisen. Somit bleibt die dritte übrig: Kann demnach eine Intuition vorhanden sein, der man aber keinen Status als Grund zubilligen sollte?

Bei genauer Betrachtung muss diese Möglichkeit in zwei weitere Möglichkeiten aufgespalten werden:Der erste Fall tritt etwa dann auf, wenn man sich die Golfspielerin als Laien vorstellt: Aufgrund ihrer mangelnden Erfahrung kann, selbst wenn die Spielerin eine Intuition in Bezug auf die Wahl des Eisens hat, diese Intuition kaum als Grund für eine Überzeugung oder Handlung gelten. Zu groß ist die Wahrscheinlichkeit, dass die Intuition aufgrund kognitiver Verzerrungen oder anderer irrelevanter Faktoren zustande gekommen ist. Im zweiten Fall hingegen wird grundsätzlich verneint, dass Intuitionen Gründe sein können; also auch, wenn die Golfspielerin Expertin auf ihrem Gebiet ist. 
Jemand, der dieser Ansicht ist, würde sie wahrscheinlich damit begründen, dass eine Intuition nicht im selben Maß verfügbar ist wie etwa eine Überzeugung oder Wahrnehmung, was aber für eine Gründe-Relation notwendig wäre.

Diese Varianten der Möglichkeit, wonach Intuitionen keine Gründe liefern, sind ernsthaft zu prüfen. Gegen beide werde ich im Folgenden vertreten, dass Intuitionen rechtfertigende Kraft haben und somit Gründe sein können. Die erste Möglichkeit betreffend ist dies insofern problematisch, als Intuition fehlbar ist. In vielen Fällen scheint Intuition in die Irre zu führen. Diese Fälle müssen im Rahmen einer Theorie der intuitiven Rechtfertigung erklärt werden können. Das Streben nach einer solchen Erklärung wird uns zunächst zu einer externalistischen Perspektive führen, aus der quasi von außen auf das Subjekt schauend festgestellt wird, inwiefern dessen intuitive Überzeugungen gerechtfertigt sind. Hierbei wird das Konzept der Adaptation bedeutsam sein. Die externalistische Perspektive wird sich jedoch als nicht hinreichend für eine Theorie der intuitiven Rechtfertigung erweisen, da die internalistische Forderung ernst genommen werden muss, wonach Gründe immer auch Gründe für das Subjekt sein müssen. ${ }^{184}$

Hiermit sind wir bei der zweiten Möglichkeit angelangt, wie die These vertreten werden kann, wonach Intuitionen keine Gründe sein können. Diese Möglichkeit wird scheinbar dadurch plausibilisiert, dass intuitive Überzeugungen als auf einem maßgeblich nichtpropositionalen Fundament ruhend beschrieben wurden. Somit stellt sich das Problem der nichtpropositionalen Rechtfertigung. Nach einer verbreiteten Ansicht können Gründe nur dann Gründe für ein Subjekt sein, wenn sie propositional und somit durch die Begriffe des Subjekts konstituiert sind. Die Lösung dieses Problems wird darin bestehen, die Verknüpfung zwischen Begründung und Begrifflichkeit zu hinterfragen und ein Modell nichtbegrifflicher Rechtfertigung in Bezug auf Intuition zu entwerfen, welches dennoch der internalistischen >Grund-fürsSubjekt<-Erfordernis Rechnung trägt. Schließlich sollen die externalistische und internalistische Perspektive zu einem moderaten Externalismus der intuitiven Rechtfertigung, der die >Grund-fürs-Subjekt<-Forderung als notwendige, jedoch nicht hinreichende Bedingung annimmt, verbunden werden. Insgesamt soll dadurch aufgezeigt werden, inwiefern wir berechtigt sind, Überzeugungen mit Verweis auf unsere Intuition zu begründen.

184 Dieser Gedanke fußt auf der kaum bestreitbaren Annahme, dass Rechtfertigung eine personenrelative Eigenschaft ist (vgl. Grundmann 2008, S. 229). Der Übergang von dieser Annahme zu einem Internalismus der epistemischen Rechtfertigung ist allerdings kontrovers. 
Die Frage der intuitiven Rechtfertigung stellt sich also genauer gesprochen in Bezug auf intuitive Überzeugungen. ${ }^{185}$ Hier lassen sich aufgrund der Zweistelligkeit des Rechtfertigungsprädikats zwei Fragen stellen: Erstens, ist die intuitive Überzeugung gerechtfertigt? Zweitens, rechtfertigt die intuitive Überzeugung andere Überzeugungen? Der zweite Fall ist auf den ersten reduzierbar: Die intuitive Überzeugung kann genau dann andere Überzeugungen rechtfertigen, wenn sie ihrerseits gerechtfertigt ist. Somit lässt sich die Frage der Rechtfertigung auf die Rechtfertigung der intuitiven Überzeugung durch das entsprechende nichtpropositionale, oft ebenfalls >Intuition< genannte Fundament eingrenzen, wodurch sich die Anschlussfrage ergibt, wie nichtpropositionale Gehalte rechtfertigende Kraft haben können. Wenn im Folgenden der Einfachheit halber von Intuitionen und intuitiver Rechtfertigung gesprochen wird, ist damit, wenn nicht anders angegeben, stets diese spezifische Rechtfertigungsbeziehung gemeint, die sich im Wesentlichen zwischen Intuitionen $_{\mathrm{NP}}$ und intuitiven Überzeugungen befindet.

Außerdem ist zu präzisieren, was im Folgenden unter >Grund < verstanden werden soll. Aus den obigen Ausführungen folgt bereits, dass es um Gründe im epistemischen Sinn geht; d.h., die hier interessierenden Gründe haben einen Bezug zur Erkenntnis. Nun kann noch weiter fokussiert werden: Es geht um Gründe, durch die das Subjekt die entsprechende Überzeugung hat. Das Subjekt stellt seine Überzeugungen auf dieser Art der Gründe ab. In der englischen Literatur spricht man daher von der grounding relation oder basing relation:

Here, $\mathrm{S}$ believes $\mathrm{p}$ on the basis of the reason. The belief that $\mathrm{p}$ thus stands in the (epistemic) basis (or basing) relation to the reason, which is a ground on account of which $S$ believes p. $^{186}$

Zur Illustration und Abgrenzung der basing relation ein Beispiel: Nehmen wir an, jemand ist allergisch gegen Nüsse, wobei die Person selber keine Kenntnis über ihre Allergie hat. Wir können dann in einer verbreiteten Verwendungsweise von >Grund sagen: Die Person hat einen Grund, keine Nüsse zu essen.

\footnotetext{
185 Intuitionen können auch - wie etwa im Golf-Beispiel ersichtlich wurde - Gründe für Handlungen sein. Jedoch werde ich dem Verhältnis zwischen Intuitionen und Handlungen nicht weiter nachgehen, sondern auf das rechtfertigende Verhältnis zwischen Intuitionen $_{\mathrm{NP}}$ und intuitiven Überzeugungen fokussieren. Bei der Diskussion um Handlungsgründe könnten Komplikationen ins Spiel kommen, die von der zentralen Frage der nichtbegrifflichen Rechtfertigung zunächst ablenken würden.

186 Audi (1998, S. 128).
} 
Dies ist jedoch kein Grund im Sinn der basing relation, denn die Person stellt keine ihrer Überzeugungen darauf ab. ${ }^{187}$

Was kann den Status eines Grundes im Sinn der basing relation erhalten? Hier sind die Positionen breit gefächert: Viele wollen nur Überzeugungen den Status als Gründe zubilligen, andere lassen auch Erfahrungsgehalte zu, wobei hier ein weiterer Streitpunkt ist, ob diese begrifflich erfasst werden müssen, um Gründe zu sein. Auf diese Fragen wird zurückzukommen sein. Bereits hier kann festgehalten werden, dass das im Folgenden vorausgesetzte Verständnis von $>$ Grund $<$ keineswegs nur Überzeugungen bzw. $>$ Grund-Propositionen $<188$ als Gründe für Überzeugungen zulässt; ansonsten könnten Intuitionen ${ }_{\mathrm{NP}}$ per definitionem keine Gründe sein. Vielmehr scheint plausibel, dass zwei Bedingungen für eine basing relation notwendig sind: Zum einen muss der Grund die Ursache oder zumindest eine der Ursachen für die Überzeugung sein, zum anderen muss der Grund in normativer Relation zur Überzeugung stehen, sodass der Grund das Subjekt dazu berechtigt, die Überzeugung zu haben. ${ }^{189} 190$ Im Folgenden werde ich die Auffassung vertreten, dass Intuitionen $\mathrm{NP}_{\mathrm{im}}$ genannten Sinn Gründe für intuitive Überzeugungen sein können.

\subsection{Externalistische Rechtfertigung: Fallibilität und Reliabilität der Intuition}

»Ich möchte gerne meinen Intuitionen folgen, aber meistens fühle ich mich zu unsicher, um dies zu tun.« Dies äußerte eine Freundin mir gegenüber. Im weiteren Gespräch ergab sich, dass meine Freundin in der Intuition eine

\footnotetext{
187 Für das Beispiel und eine kritische Frage, die zu dieser Präzisierung geführt hat, danke ich Julien Dutant.

188 Vgl. Audi (1998, S. 128).

189 Aufgrund des zweiten Kriteriums sollten Gründe sorgfältig von Ursachen unterschieden werden (vgl. Kambartel 2005).

190 Für eine weitere Unterscheidung und Kritik verschiedener Verständnisse von >Grund und >Begründung< siehe Spohn (2001, 2018). Wolfgang Spohns eigener Vorschlag der >Positiven-Relevanz-Relation< ist, dass A genau dann ein Grund für B ist, »wenn A den Glauben an B stärkt, d.h., wenn der Glaubensgrad von B unter der Bedingung A höher ist als unter der Bedingung non-A, d.h. wenn A für B positiv relevant ist« (Spohn 2001, S. 48). Sobald man aber, wie auch hier vorgeschlagen, von der basing relation-Konzeption von Gründen ausgeht, scheint Spohns Gründe-Kriterium nicht hinreichend, da Überzeugungen positiv relevant für andere Überzeugungen sein können, ohne dass sie Gründe im Sinn der basing relation sind. Darüber hinaus ist Spohn ein Vertreter der in dieser Arbeit zurückgewiesenen Auffassung, wonach Gründe propositional verfasst sein müssen (vgl. Spohn 2018, S. 3839f.).
} 
Erkenntnisquelle sieht, die dem diskursiven Denken ebenbürtig oder gar überlegen sein kann. Gleichzeitig jedoch zweifelt sie an der Zuverlässigkeit dieser Erkenntnisquelle. Wie lassen sich diese scheinbar einander zuwiderlaufenden Einschätzungen erklären?

Ein entsprechendes Spannungsfeld lässt sich auch zwischen den vorherrschenden philosophischen und psychologischen Positionen zur Intuition erkennen: Einerseits wurde Intuition zur höchsten Form der Erkenntnis erhoben, die alleine unfehlbare Einsichten ermögliche (man denke etwa Spinozas scientia intuitiva), andererseits wurde Intuition als stark anfällig für kognitive Verzerrungen und dadurch irreführend dargestellt (v.a. in Kahnemans heuristics and biases-Ansatz). ${ }^{191}$ Die Fallibilität der Intuition wurde im vorigen Kapitel gegen einige klassische Positionen bereits anerkannt; doch bleibt die Frage, wie zuverlässig Intuitionen zu Erkenntnis führen und auf welche Art die Rechtfertigung erfolgt. So wäre Intuition, falls sie in den meisten Fällen irreführend ist, sicherlich keine valable Quelle der Erkenntnis.

Ein erster, oft gangbarer Weg zur Beurteilung der Zuverlässigkeit der Intuition in einem gegebenen Fall ist die deduktive Nachprüfung. Nehmen wir als Beispiel eine Wissenschaftlerin, die lange über die Lösung eines Problems nachgedacht hat, ohne Fortschritte gemacht zu haben. Nun hat sie eine Intuition, wie sie das Problem angehen kann, beispielsweise in Form eines neuen Experimentaufbaus. Nehmen wir weiter an, dieses neue Experiment führe zum Erfolg. Die Wissenschaftlerin wird nach einer Erklärung suchen, warum sich der Erfolg eingestellt hat. Sie kann also ihre Intuition durch deduktive Schritte nachprüfen. Wir stellen in diesem Fall, der uns auch im Alltag vertraut ist, eine Rollenteilung zwischen Intuition und diskursivem Denken fest: Intuition für die Heuristik und das Entdecken, diskursives Denken für die Logik und das Nachprüfen. Diese Rollenteilung lässt sich gut mit den Typen 1 und 2 des menschlichen Denkens, wie sie durch Dual-Prozess-Theorien beschrieben wurden, erklären: Typ 1-Denken eignet sich aufgrund seiner Geschwindigkeit und hohen Kapazität der Informationsverarbeitung für das heuristisch-entdeckende Forschen, Typ 2-Denken für das sichernde, explizit machende Nachprüfen.

In erkenntnistheoretischer Hinsicht wird die Frage nach der Zuverlässigkeit der Intuition erst aufgrund derjenigen Fälle interessant, in denen eine

191 Vgl. Kahneman (2012). Es würde aber zu kurz greifen, dies als einen Gegensatz zwischen Philosophie und Psychologie darzustellen. Einerseits gibt es bedeutende Intuitionsskeptiker in der Geschichte der Philosophie; es sei an Immanuel Kants Reduktion der Intuition auf empirische Anschauung erinnert. Andererseits wurde auch in der psychologischen Forschung der Erkenntniswert der Intuition betont und durch Studien erhärtet (vgl. Gigerenzer 2007; Klein/Calderwood/Clinton-Cirocco 2010). 
deduktive Nachprüfung nicht oder nur schwer möglich ist: Inwiefern dürfen wir uns in diesen Fällen auf die Intuition verlassen? Hier müssen wir die $\mathrm{Zu}$ verlässigkeit der Intuition als Erkenntnisquelle abschätzen können, wobei zunächst unklar ist, welche Referenzpunkte wir hierfür nehmen sollen, was auch im Beispiel meiner Freundin deren Unsicherheit erklärt. Eine Hypothese in Bezug auf möglichen Referenzpunkte soll im Folgenden entwickelt werden. Diese Hypothese ließe sich erst im Rahmen umfangreicherer, empirisch unterstützter Arbeiten weiter prüfen. Hier geht es lediglich darum aufzuzeigen, dass eine plausible Erklärung der Zuverlässigkeit der Intuition möglich ist.

Die Hypothese zur Zuverlässigkeit der Intuition, die im Folgenden plausibilisiert werden soll, bezieht sich auf die Entstehungsgeschichte der jeweiligen Intuition, und zwar auf deren Adaptation:

(AH): Intuitionen haben sich als Bestandteil der Kognition von Subjekten entwickelt, insoweit sie für diese Subjekte adaptiv sind. ${ }^{192}$

>Adaptation<, bzw. >adaptiv< soll hier in Anlehnung an evolutionsbiologische und psychologische Konzepte verstanden, jedoch nicht auf einen bestimmten Bereich (beispielsweise auf genetische Adaptation) eingeschränkt werden. Grundsätzlich bezeichnet Adaptation die Veränderungen eines Organismus, die dazu tendieren, sein Leben in dieser Umwelt zu sichern oder zumindest zu erleichtern. ${ }^{193}$ Das maßgeblich evolutionsbiologisch geprägte Konzept der Adaptation lässt sich mit eher psychologischen Verständnissen verbinden: Adaptation kann demzufolge eine Eigenschaft der Struktur, der Physiologie oder des Verhaltens eines Organismus bezeichnen, die dazu beiträgt, ein Problem im Leben dieses Organismus zu lösen. ${ }^{194}$ Im Hinblick auf Intuition bringt (AH) zum Ausdruck, dass eine jeweilige Intuition besteht, weil sie sich als adaptiv in Bezug auf das Leben eines Subjekts bzw. auf die Leben von dessen Vorfahren herausgestellt hat. Es wird also auf die Entstehungsgeschichte der Intuition Bezug genommen, um das Bestehen derselben zu erklären und im Anschluss daran auch deren Reliabilität zu beurteilen, wobei es sich bei dieser Geschichte einerseits um die Biografie eines Individuums,

192 Für den Hinweis darauf, dass das Konzept der Adaptation für eine Theorie der intuitiven Rechtfertigung bedeutsam sein könnte, danke ich Mu Lin.

193 Vgl. Hine/Martin (2016).

194 Vgl. Colman (2015). 
andererseits um eine phylogenetische Geschichte (in Bezug auf die Evolution der menschlichen Kognition) handeln kann. ${ }^{195,196}$

(AH) soll zunächst direkt auf ein Beispiel irreführender Intuition angewandt werden, da diese die Frage nach der Reliabilität der Intuition aufgeworfen hat. Kommen wir hierzu auf das Beispiel des Spielerfehlschlusses zurück. Grundsätzlich haben Menschen die Intuition, dass etwas, das sich häufig oder kürzlich ereignet hat, sich wahrscheinlicher wieder ereignen wird als etwas, das sich weniger häufig oder vor längerer Zeit ereignet hat. Nennen wir diese im Folgenden $>$ Wiederholungsintuition<. Aufgrund der Wiederholungsintuition hätte ein Spieler grundsätzlich die Erwartung, dass sich eine bereits sehr oft gewürfelte Zahl mit höherer Wahrscheinlichkeit beim nächsten Wurf wieder zeigen wird. Unter dem Eindruck der Wiederholungsintuition würde der Spieler somit wahrscheinlich zu hohe Beträge auf die Zahlen wetten, die in den letzten Runden überdurchschnittlich häufig gewürfelt wurden.

Wiederholungsintuitionen sind in den meisten Fällen erfolgreich und haben sich als adaptiv herausgebildet, weil in diesen Fällen kausale Bezüge zwischen den vergangenen und zukünftigen Ereignissen in Bezug auf ein bestimmtes Objekt bestehen: Beispielsweise wird der Stier, wenn ich ihm erneut das rote

195 In Bezug auf die maßgeblich durch die experimentelle Philosophie ausgelöste Diskussion um die Kulturrelativität der Intuition ließen sich wohl auch Ebenen der Adaptation zwischen Individuum und Menschheit ausmachen; z.B. Adaptation in Bezug auf das Leben einer bestimmten Gruppe oder kulturellen Gemeinschaft. Trifft es zu, dass Intuition in diesem Sinn kulturrelativ ist, wäre dies in den hier vorgestellten Ansatz integrierbar. Um die Ausführungen nicht unnötig zu komplizieren, verzichte ich an dieser Stelle auf die Einbindung der kulturellen Ebene.

196 Eine Erklärung der Rechtfertigung, die sich wie die vorliegende auf Adaptation stützt, kann dem Vorwurf ausgesetzt sein, die epistemologisch zentrale Unterscheidung zwischen Genese und Geltung zu vernachlässigen. Während `Genese`Gründe des Fürwahrhaltens bezeichnet, steht >Geltung \& für Gründe des Wahrseins (vgl. Schildknecht/Teichert/van Zantwijk 2008). Diesem Vorwurf zufolge würde hier ein unreflektierter Übergang von einer genetischen Erklärung der Intuitionen zu einer epistemologischen Rechtfertigung derselben vorgenommen.

Dem genannten Vorwurf kann auf zwei Weisen begegnet werden: Erstens kann die Trennung zwischen Genese und Geltung hinterfragt werden, etwa im Sinne eines teleosemantischen Ansatzes, demzufolge die Genese der Repräsentationen für ihre Geltung entscheidend ist (siehe unten). Zweitens kann aber auch - ohne solche naturalistische Verpflichtungen einzugehen - der heuristische Weg beschritten werden: De facto werden in einer normalen Umgebung öfter Dinge für wahr gehalten, die tatsächlich wahr sind. Ein Blick auf die Gründe des Fürwahrhaltens einer Intuition und die Beurteilung, ob ein gegebener Fall der Intuition zu diesen Gründen passt, kann daher einen Hinweis darauf liefern, wie wahrscheinlich die Intuition der Wahrheit entspricht. Dies entspricht einem minimalen Externalismus und reicht somit für die vorliegende Arbeit aus, in welcher internalistische Elemente die Theorie der Rechtfertigung vervollständigen werden. 
Tuch zeige, wahrscheinlich wieder rasend werden, wie die vielen Male, in denen ich dies zuvor beobachtet habe. Natürlich kann auch in solchen Fällen das erwartete Ereignis einmal nicht eintreten, doch im Normalfall ist es zu erwarten, und daher ist die Wiederholungsintuition adaptiv.

Nun gibt es jedoch eine oder mehrere signifikante Gruppen von Fällen, in denen diese Intuition in die Irre geht. Als >signifikant $<$ sind diese Gruppen insofern zu bezeichnen, als sie entweder eine hinreichend große Anzahl von Fällen oder hinreichend bedeutsame (z.B. überlebenswichtige) Fälle umfassen; als $>$ Gruppen<, insofern diese Fälle durch eine gemeinsame Charakteristik miteinander verbunden sind, und zwar im Normalfall dadurch, dass sie sich auf dasselbe Objekt oder dieselbe Situation beziehen. Dies ist in unserem Fall ein Ereignis mit mehreren möglichen Ausgängen, deren Auftretenswahrscheinlichkeit nicht von früheren Häufigkeiten bestimmt wird. In Bezug auf die signifikante Gruppe von Fällen hat sich eine gegenläufige Intuition aufgrund ihrer Adaptation herausgebildet. Diese Intuition kann als >sekundäre Intuition< bezeichnet werden, insofern sie die primäre Intuition im Sinne einer Überspielung oder Ersetzung korrigiert. In unserem Beispiel ist es die sekundäre Intuition des Spielerfehlschlusses, welche die primäre Intuition der Wiederholung korrigiert. ${ }^{197}$

Die Erklärung der Spielerfehlschluss-Intuition auf der Basis von (AH) provoziert den folgenden Einwand: Der Spielerfehlschluss korrigiert die Wiederholungsintuition im vorliegenden Fall nicht, vielmehr wird hier ein Fehler durch einen anderen Fehler überspielt oder ersetzt. Die Intuition des Spielers erfasst die korrekten Wahrscheinlichkeiten weder im primären noch im sekundären Fall. Wie kann also in diesem Zusammenhang berechtigt von >Korrektur gesprochen werden? Als Antwort auf diese kritische Frage kann darauf verwiesen werden, dass der Anspruch generell unberechtigt ist, wonach sich mit einer sekundären Intuition jeder Fehlschluss vermeiden lässt. Einzelfälle sind immer noch möglich, in denen die sekundäre Intuition nicht korrekt ist, solange diese nicht die Schwelle einer signifikanten Gruppe im oben geschilderten Sinn erreichen. Mit (AH) verbunden ist also die Annahme, dass die korrektive Intuition in einer hinreichenden Anzahl von Fällen für das Leben des Organismus von Vorteil ist. Dies ist im vorliegenden Beispiel

197 Denkbar wäre auch, den Spielerfehlschluss direkt durch die Adaptation einer primären Intuition zu erklären: Möglicherweise bildet das Subjekt hier eine Analogie zu begrenzten Ressourcen: Ist viel davon aufgebraucht (die Zahl wurde oft gewürfelt), bleibt nur wenig übrig (die Zahl wird kaum wieder gewürfelt werden). Eine weitere primäre Intuition, die dem Spielerfehlschluss zugrunde liegen könnte, ist die »Tendenz zur Mitte«: Weil gleiche Wahrscheinlichkeit zur Erwartung gleicher Häufigkeit führt, wirkt sich eine hier problematische Durchschnittsbildung auf die Erwartung des nächsten Wurfs aus. 
plausibel: Insofern die sekundäre Intuition im Fall der kausal nicht miteinander verbundenen Situationen >wird nicht wieder auftreten $<$ als Heuristik hervorbringt, gibt sie dem Denken und Handeln des Individuums generell eine negative Richtung hin zum Verzicht auf eine Handlung oder zur Vermeidung einer Situation.

Zur Veranschaulichung des bisher Gesagten ein Beispiel: Wenn eine Jägerin viele oder bedeutende Male das Anfertigen eines Talismans als hilfreich für den Jagderfolg erlebt hat, wird sie gemäß der primären Intuition der Wiederholbarkeit diesen Talisman wieder anfertigen. Nun kann die sekundäre Intuition diese primäre Intuition insofern korrigieren, als andere Typ 1-Prozesse aufgrund relevanter Informationen dazu in der Lage sind, die beiden Ereignisse als kausal nicht miteinander verbunden zu identifizieren. S wird gemäß der sekundären Intuition also keinen Talisman anfertigen und dadurch Energie sparen. Demgegenüber sind Fälle wie das Würfelspiel, in denen es um die Wahrscheinlichkeit des Auftretens von sechs oder mehr verschiedenen Ereignissen geht, bedeutend komplizierter und in Bezug auf Häufigkeit und Wichtigkeit von zu geringer Bedeutung, als sie die Adaptation dieser sekundären Intuition einschränken könnten. Somit bildet sich die entsprechende Intuition heraus.

Als adäquat erweist sich ( $\mathrm{AH})$ auch in Bezug auf die Erklärung subjektspezifischer Intuitionen, wie im Beispiel der Feuerwehrkommandantin Claire. Hier ist die Entstehungsgeschichte in Bezug auf die Biografie Claires heranzuziehen. Auf diese Weise entstehen und verfestigen sich Intuitionen auch auf der Ebene des Subjekts durch Adaptation. Die Intuition in Bezug auf die Einsturzgefahr des brennenden Gebäudes hat sich aufgrund der mannigfaltigen Erfahrung Claires herausgebildet. Einerseits gab ihr diese Erfahrung die Gelegenheit, immer wieder die entsprechenden Erfahrungsgehalte aufzunehmen und vorrangig innerhalb des Typ 1-Denkens miteinander in Bezug zu setzen. Andererseits entsprechen die in vielen Übungssituationen wiederholt gestellten Anforderungen Problemstellungen, für welche die Herausbildung der Intuition eine adäquate Lösung bietet: Mittels Intuition kann schneller die Gesamtsituation erfasst, mit Wissen und Erfahrung abgeglichen und auf diesen Grundlagen passend entschieden werden.

Auch zu den Cartesischen Intuitionen passt (AH). Um sich dies vor Augen zu führen ist zu bedenken, dass diese Intuitionen von Grundlagen des menschlichen Denkens handeln. Selbstevidente Wahrheiten wie sein Dreieck hat drei Seiten oder der Satz vom ausgeschlossenen Widerspruch machen eine deduktive Herleitung entweder überflüssig oder sogar unmöglich; zugleich sind diese Wahrheiten Fundamente des menschlichen Argumentierens. Ihre Akzeptanz ist die Voraussetzung für die Möglichkeit, weiteren Argumentationen 
folgen zu können. ${ }^{198}$ Insofern haben sich Intuitionen in Bezug auf die menschliche Kognition vermutlich als adaptiv erwiesen, wenn sie die Ausdifferenzierung eines argumentativen Systems ermöglichten. Ähnliches lässt sich in Bezug auf die Intuition sich denke, ich existiere< sagen. Beispielsweise ermöglicht das Bewusstsein des eigenen Denkens eine Reflexion über dieses, was auch in alltäglichen Situationen bedeutsam ist (etwa dann, wenn man sich überlegt, ob man in einer Position ist, etwas mit genügend hoher Sicherheit annehmen zu dürfen). Das Bewusstsein der eigenen Existenz wiederum ist zentral für die conditio humana und wohl auch für gewisse Tiere; es geht somit einher mit der Evolution des menschlichen Denkens.

Nun soll noch etwas mehr zum Hintergrund der hier vertretenen Hypothese (AH) gesagt werden. $(\mathrm{AH})$ ist von Ruth Millikans teleologischer Theorie des mentalen Gehaltes inspiriert. ${ }^{199}$ Generell versuchen teleologische Theorien des mentalen Gehaltes den Gehalt mentaler Repräsentationen durch Bezugnahme auf eine teleologische Konzeption von Funktion zu erklären. ${ }^{200}$ In diesem Sinn hält Millikan fest:

[...] what makes a thing into an inner representation is, near enough, that its function is to represent. But, I shall argue, the way to unpack this insight is to focus on representation consumption, rather than representation production. It is the devices that use representations which determine these to be representations and, at the same time (contra Fodor), determine their content. ${ }^{201}$

Die teleologische Konzeption ist hier, dass jeder der folgenden drei Bestandteile Funktionen hat, wobei unter Funktion >biologischer Zweck zu verstehen ist: Das Gehalte produzierende System hat die Funktion, die Repräsentation zu produzieren; die Repräsentation hat die Funktion, sie zu repräsentieren; das konsumierende System hat die Funktion, die Repräsentation zu konsumieren. Die Systeme haben eine >natürliche Geschichte`, in welcher sie sich dazu entwickelt haben, eine Repräsentation so zu repräsentieren, bzw. zu konsumieren, wie sie dies tun; dies entspricht der Adaptation. In diesem Sinn wird der Gehalt einer Repräsentation nicht in erster Linie durch einen direkten, etwa kausalen

198 Daraus folgt nicht, dass alle Menschen auf diese selbstevidenten Grundlagen des Denkens und der Logik in der Form von Überzeugungen Zugriff haben. Es ist wahrscheinlich, dass solche selbstevidenten Wissensgrundlagen vielen Menschen bekannt sind, ohne dass diese bei ihnen die Form propositionaler Überzeugungen annehmen. Eine ausführliche Auseinandersetzung mit der Bekanntschaftsrelation wird in Kap. 4.5 folgen.

199 Vgl. Millikan (1989a, 1989b).

200 Vgl. Neander (2012).

201 Millikan (1989a, S. 283f.). 
Einfluss der Außenwelt bestimmt, sondern aufgrund der Art und Weise, wie sich das die Repräsentation produzierende sowie konsumierende System an die Umwelt angepasst haben - oder evolutionsbiologisch ausgedrückt: aufgrund dessen, wofür sie evolutionär selektiert wurden.

Der Adaptations-Hypothese über Intuition liegt ebenfalls die Idee zugrunde, dass der Gehalt einer Intuition durch die Funktion bestimmt wird, welche diese Intuition hat - nämlich, etwas zu repräsentieren - sowie durch die Funktion der Systeme, welche diese Intuition konsumieren. Im Fall einer inneren Repräsentation (im Unterschied zum Fall der Kommunikation) sind sowohl die produzierenden als auch die konsumierenden Systeme Teile des repräsentierenden Organismus. ${ }^{202}$ Im Fall der Intuition sind verschiedene >Konsumenten< vorhanden: Einer davon ist das Typ 2-Modul, welches intuitive Überzeugungen generiert. Ein anderes konsumierendes System ist vermutlich das Modul (bzw. mehrere Module), das von der Intuition direkt zu Handlungen führt, ohne den Umweg über intuitive Überzeugungen zu gehen. In jedem Fall ist - Millikans Theorie entsprechend - der Gehalt der Intuition durch die einwandfreie Funktion (proper function) der konsumierenden Systeme bestimmt. Somit können wir mit Millikan präziser beschreiben, was Adaptation in Bezug auf Intuition bedeutet und dabei auch Fehlrepräsentation miteinschließen: Fehlerhafte Intuitionen sind in einer teleologischen Theorie des mentalen Gehaltes gut integrierbar, da sie als Ausnahmen von funktionalen Intuitionen fungieren. Funktionale Intuitionen behandeln signifikante Gruppen von Repräsentationen erfolgreich, auf Kosten von Fehlern in Bezug auf Repräsentationen, die von der Gruppenregel abweichen. Wird die Gruppe der Fehlrepräsentationen signifikant, bildet sich - entsprechend der Konzepte der Funktionalität und Adaptation - eine sekundäre Intuition aus, die korrigierend wirkt.

Ein für die Frage der Rechtfertigung entscheidender Aspekt des teleofunktionalen Ansatzes ist, dass sich ein Graben zwischen Funktionalität und Wahrheitsorientierung bilden kann. Gemäß $(\mathrm{AH})$ bilden sich Intuitionen heraus, weil sie adaptiv sind, aber dies bedeutet nicht, dass sie in jedem Fall die Wahrheit repräsentieren. Auf Basis von ( $\mathrm{AH}$ ) lässt sich in Bezug auf die gestellte Frage, inwiefern Intuitionen zuverlässig sind, nun Weiteres präzisieren. Wann dürfen wir unseren Intuitionen trauen? Grundsätzlich können sie immer in die Irre führen, zugleich bilden sie oft wertvolle oder gar unentbehrliche Wege zu neuer Erkenntnis. Wenn wir nicht die Möglichkeit haben, die Intuitionen deduktiv zu überprüfen, sollten wir aufgrund von $(\mathrm{AH})$ die folgenden beiden Faustregeln beachten:

202 Vgl. Neander (2012). 
Erstens sollten wir berücksichtigen, ob wir im relevanten Gebiet Expertise haben. So kann Claire ihrer Intuition in Bezug auf das brennende Gebäude grundsätzlich trauen, weil sich durch ihre große Erfahrung im relevanten Gebiet diese Intuitionen als adaptiv herausbilden konnten. Hingegen ist statistisches Wissen in Alltagszusammenhängen oft wenig bedeutsam und wird vor allem zugunsten von Heuristiken zurückgestellt.. ${ }^{203}$ Der Hintergrund entsprechender intuitiver Fehler ist stets, dass sich Intuitionen als Formen der Typ 1-Kognition nicht durch Präzision, sondern durch Leistungsfähigkeit auszeichnen, wodurch sie gemäß (AH) erfolgreich genug sind, um sich trotz der mit ihnen verbundenen Fehlrepräsentationen zu festigen. Zwar ist bekannt, dass Experten auch im Bereich ihrer Expertise fehlerhafte Intuitionen haben und diesen oft folgen. ${ }^{204}$ Dies widerspricht aber dem soeben Ausgeführten nicht, sondern wird als Überlagerung des Experten-Wissens (allenfalls auch der Experten-Intuitionen) durch die allgemein-menschlichen Intuitionen erklärbar, die sich trotz Expertise im relevanten Feld als stärker erweisen können. Immerhin bleibt in aller Regel der Weg offen, der weiter oben angesprochen wurde: die durch deduktives Typ 2-Denken erfolgende Überprüfung der intuitiven Erkenntnis bzw. Fehlrepräsentation.

Als zweite Faustregel in Bezug auf die Zuverlässigkeit unserer Intuitionen sollten wir berücksichtigen, wie fatal es wäre, wenn wir eine fehlerhafte Intuition als intuitive Überzeugung übernehmen und aufgrund dieser wohl auch handeln würden. Gegebenenfalls kann dieses Risiko gegen den Vorteil abgewogen werden, der entsteht, wenn wir der entsprechenden Intuition folgen und sie sich als richtig herausstellen würde. Hierzu ein Beispiel: Ich komme bei einer Wanderung an eine Kreuzung und habe die intuitive Überzeugung, dass ich dem Pfad zu meiner Linken folgen sollte. Normalerweise ist es gemäß der Faustregel gut, dieser Intuition zu folgen, da nichts voraussehbar Schlechtes dadurch geschehen kann und wohlmöglich in meinem Typ 1-Denken Informationen verarbeitet wurden, die tatsächlich gute Gründe für die Wahl des linken Pfads konstituieren. Als kontrastierendes Beispiel lässt sich der Fall eines Personalverantwortlichen anführen, der eingegangene Stellenbewerbungen durchsieht. Wenn der Personalverantwortliche bestimmte Intuitionen hat, eine bestimmte Bewerberin einer anderen vorzuziehen, sollte er bezüglich seiner eigenen Intuition kritisch sein, weil hier ein Irrtum gravierend ist. Beispielsweise könnte er ohne weiteres einer diskriminierenden kognitiven Verzerrung unterliegen, die aufgrund des ausländisch klingenden

203 Vgl. Gigerenzer (2007, Kap. 6).

204 Vgl. Tversky/Kahneman (1974). 
Namens einer Kandidatin entsteht. ${ }^{205}$ Andererseits kann er aufgrund seiner Erfahrung sehr gute Intuitionen in Bezug auf die Auswahl von Kandidatinnen haben. Doch die mögliche Überlagerung der Experten-Ebene durch die Ebene der >menschlichen Intuitionen< darf nicht außer Acht gelassen werden. Auch Experten haben, wie im Beispiel des Personalverantwortlichen, oftmals die persönliche oder gar offizielle Pflicht, ihre Intuitionen deduktiv nachzuprüfen.

\subsection{Internalistische Rechtfertigung als notwendige Ergänzung}

Einleitend wurde die Frage gestellt, inwiefern intuitive Überzeugungen gerechtfertigt sind. Haben die Ausführungen in Bezug auf die Reliabilität der Intuitionen diese Frage zufriedenstellend beantwortet? Einerseits, aus unserer Außenperspektive auf das erkennende Subjekt, möglicherweise ja: Insofern es bei der Frage nach der epistemischen Rechtfertigung darum geht, wie das Rechtfertigende zum Finden der Wahrheit beiträgt, haben wir eine Erklärung entworfen, die auch die möglichen Fehler der Intuition einschließt. Andererseits, aus der Perspektive des Subjekts, wohl nein: Die oben skizzierte Erklärung muss dem erkennenden Subjekt nicht zugänglich sein. Die Forderung, das Subjekt müsse sich der Adaptationserklärung bewusst sein, um in der entsprechenden intuitiven Überzeugung gerechtfertigt zu sein, ist unhaltbar. Zwar wurden Faustregeln erwähnt, die in Bezug auf Intuition befolgt werden können, und auch die deduktive Nachprüfung von Intuitionen wurde als Möglichkeit ins Spiel gebracht. Weiter kann fallweise gefordert werden, dass diese Regeln befolgt werden müssen, damit eine Intuition gerechtfertigt ist. Dagegen wäre die Forderung, wonach ein Subjekt diese Regeln nicht nur befolgen, sondern sich der Befolgung und Begründung der Regeln auch bewusst sein muss, gewiss überzogen. Daher fordern epistemologische Internalisten wie Laurence BonJour in Bezug auf Rechtfertigung eine ganz andere Art von Gründen, nämlich solche, die dem Subjekt selber zugänglich sind:

In first approximation, an epistemological theory counts as internalist if and only if it requires that all of the elements needed for a belief to satisfy this condition must be cognitively accessible to the person in question; and as externalist, if it allows that at least some of these elements need not be thus accessible, allowing them to be external to the believer's cognitive perspective. ${ }^{206}$

205 Vgl. Bertrand/Mullainathan (2004).

206 Bonjour (2002, S. 234). 
Ein Argument für die internalistische These, wonach für eine gelingende Rechtfertigung alle Gründe dem Subjekt selber zugänglich sein müssen, erwächst aus Beispielen, in welchen alle externalistischen Erfordernisse erfüllt sind, ohne dass man sagen würde, das Subjekt sei entsprechend gerechtfertigt:

Norman besitzt tatsächlich zuverlässige hellseherische Fähigkeiten. Er hat aber aus seiner Perspektive weder Gründe für noch gegen die Zuverlässigkeit der Hellseherei und schon gar keine Gründe dafür oder dagegen, dass er persönlich diese Fähigkeit besitzt. In einer bestimmten Situation erwirbt Norman aufgrund seiner hellseherischen Fähigkeiten die wahre Überzeugung, dass sich der amerikanische Präsident in New York aufhält. Aus seiner Perspektive gibt es keine Gründe, die für oder gegen diese Überzeugung sprechen. ${ }^{207}$

BonJour zufolge würde man in diesem Beispiel keineswegs sagen wollen, dass Norman in der Überzeugung, der Präsident halte sich in New York auf, gerechtfertigt ist. Dies wäre schlicht keine korrekte Verwendungsweise des Begriffs $>$ Rechtfertigung $<$. Externalisten - hier richtet sich die Kritik spezifisch an Vertreter des Reliabilismus - müssten aber sagen, dass Norman in seiner Überzeugung gerechtfertigt ist, da, wie wir aus der Außenperspektive wissen, seine hellseherischen Fähigkeiten die Wahrheit zuverlässig anzeigen. Daraus folgt, dass der Reliabilismus unplausibel ist. Das Beispiel lässt sich dahingehend verschärfen, dass Norman aus seiner Perspektive sogar gute Gründe hat, die gegen die Überzeugung sprechen, der Präsident halte sich in New York auf (beispielsweise, wenn im Radio die Falschmeldung verbreitet wurde, er sei heute in Washington). Wir würden Norman den Internalisten zufolge sogar als irrational ansehen, wenn er trotz dieser gegenläufigen Evidenz an der Überzeugung festhalten würde, der Präsident sei in New York. ${ }^{208}$ Daher, so die internalistische Schlussfolgerung, sind externalistische Theorien der Rechtfertigung unzureichend. Gründe müssen dem erkennenden Subjekt selber als solche zugänglich sein. Beispiele wie dasjenige Normans sollen diese Position stützen.

Das Norman-Beispiel lässt sich leicht auf den Fall der Intuition übertragen. In zahlreichen Beispielen verhält es sich ähnlich wie im Fall Normans: Das Subjekt hat eine intuitive Überzeugung, verfügt jedoch offenbar über keinerlei Gründe, die für diese Überzeugung sprechen. Selbst wenn wir die Adaptations-Hypothese als überzeugend annehmen, haben wir hier offenbar

207 Vgl. BonJour (1985, S. 41) in der Übersetzung von Grundmann (2008, S. 259f.).

208 Die Bedeutung solcher Anfechtungsründe (defeaters) ist Gegenstand einer eigenen Diskussion in der Erkenntnistheorie (defeasibility theory). Dabei wird der Verweis auf Anfechtungsgründe als Einwand gegen den Externalismus verwendet (vgl. Grundmann 2011, S. 16of.). 
eine zu Norman analoge Situation: Verfügt jemand über keine untermauernden Gründe, vielleicht sogar im Gegenteil über widerlegende Informationen, dürfte er keinesfalls die intuitive Überzeugung aufrechterhalten; mit anderen Worten: seine intuitive Überzeugung wäre nicht gerechtfertigt. In den Worten Duncan Pritchards:

[W] tend to want beliefs that are more than just safe. That is, we don't just want agents to be forming beliefs in such a way that we can rely on the truth of those beliefs, we also want agents to be cognitively responsible for their beliefs, and this is only possible if they form beliefs in ways that are responsible to the reflectively accessible grounds that they have in favour of their beliefs. ${ }^{209}$

In diesem Zusammenhang stellt sich allerdings die Frage, wie weit die Einsicht in die Gründe gehen muss, damit das Subjekt als »kognitiv verantwortlich« gelten kann. Verlangt ein Internalist hier, dass das Subjekt neben der jeweiligen Überzeugung auch die Meta-Überzeugung darüber haben muss, dass die Überzeugung auf zuverlässige Weise zustande gekommen ist? Dies würde eine internalistische Rechtfertigungserklärung der Intuition an die oben entwickelte externalistische Erklärung zurückbinden. Dem Subjekt müsste diese externalistische Erklärung zumindest in den Grundzügen präsent sein, was zweifellos unplausibel ist.

Eine externalistische Replik Thomas Grundmanns auf BonJours NormanBeispiel knüpft an diesen Punkt an: ${ }^{210}$ Grundmann zufolge dient das NormanBeispiel nicht als adäquate Kritik am epistemologischen Externalismus. Vielmehr liegt es gemäß Grundmann an einem durchwegs externalistisch erfassbaren Grund, warum wir Norman als nicht zu seiner Überzeugung gerechtfertigt erachten: Normans Überzeugung ist auf dubiose Weise zustande gekommen. Im Unterschied etwa zu Wahrnehmungsüberzeugungen bleibe völlig unklar, wie solche hellseherische Fähigkeiten funktionieren. Im Falle einer Wahrnehmungsüberzeugung, so Grundmann weiter, würden wir auch nicht zögern, dem Subjekt die Rechtfertigung dieser Überzeugung zuzusprechen, ohne dass das Subjekt Einsicht in die Zuverlässigkeit seines visuellen Apparats habe. Genau dies müssten wir aber tun, sollte das Gedankenexperiment Normans in analoger Weise für den Internalismus sprechen. Allgemeiner ausgedrückt ist, wie Robert Brandom durchaus zutreffend feststellt, »rein gar nichts unverständlich daran, Überzeugungen zu haben, für die wir keine Gründe geben können «. ${ }^{211}$ Mit dem Verweis auf Religion argumentiert

\footnotetext{
209 Vgl. Pritchard (2007, S. 184).

210 Vgl. Grundmann (2008, S. 26of.).

211 Brandom (2001, S. 139).
} 
Brandom weiter, dass, würde sich die entsprechende Glaubensüberzeugung als wahr erweisen und wäre sie auf zuverlässige Weise zustande gekommen, nichts gegen deren Einordnung als Wissen sprechen würde. Dies wäre aber nicht möglich, wenn wir an der klassischen Forderung festhalten, wonach für Wissen Rechtfertigung vorliegen muss und wir zugleich das internalistische Zugänglichkeitskriterium akzeptieren. Dieses Kriterium scheint Grundmann und Brandom zufolge also problematisch zu sein.

Als Einleitung zu einer Replik auf Grundmann und Brandom ist eine wichtige Unterscheidung zu treffen: Das internalistische Kriterium kann auf zwei Weisen verstanden werden: im Sinne einer Zugänglichkeit, oder aber im schwächeren Sinne einer Verfügbarkeit der Gründe für das Subjekt. Die Überlegungen Grundmanns und Brandoms erweisen die Zugänglichkeit zu den Gründen als unplausible Forderung, sprechen aber nicht gegen die Forderung der Verfügbarkeit. ${ }^{212}$ Auch wenn wir Pritchard darin folgen, dass die Subjekte kognitiv verantwortlich für ihre Überzeugungen sind, müssen wir nicht akzeptieren, dass dies eine Zugänglichkeit zu den Gründen voraussetzt. Ebenso kann diese kognitive Verantwortung aufgrund der bloßen Verfügbarkeit zugeschrieben werden.

Auf Grundmanns Einwand lässt sich daher im Sinne eines Verfügbarkeits-Internalismus antworten: Es stimmt zwar, dass das Subjekt keine Einsicht in die Reliabilität seiner »Überzeugungsproduktion« zu haben braucht, um eine entsprechende Rechtfertigung für seine Überzeugung zu haben. Dennoch muss der Grund ein Grund für das erkennende Subjekt sein. Dies ist im Fall der Wahrnehmungsüberzeugung durchaus gegeben. Es reicht der bloße Verweis auf die Wahrnehmung selbst; diese ist im Normalfall ein hinreichender Grund für ein Subjekt, die entsprechende Überzeugung zu bilden. (»Warum glaubst du, dass der Ball rot ist?« - »Na, weil ich das sehe.«) Ein solcher Grund ist im Fall Normans wie auch im Fall der Intuition nicht in Sicht. ${ }^{213}$ Somit bleibt es durchaus dieses Defizit, welches die Rechtfertigung verhindert, und durch welches sich ein starker Externalismus (beispielsweise in Form des Reliabilismus) als unzulänglich erweist.

212 Die hier getroffene Unterscheidung zwischen Zugänglichkeit und Verfügbarkeit ist verwandt mit der Unterscheidung `Gründe haben< vs. >Gründe geben< (having vs. giving reasons), die im Bereich der Wahrnehmung gegen McDowells Konzeptualismus ins Feld geführt wurde (vgl. Schantz 2001, S. 176f.). Möglicherweise ist es notwendig dafür, Gründe zu geben, dass diese im oben genannten Sinn zugänglich sind, jedoch nicht dafür, Gründe zu haben.

213 »Weil ich die Intuition habe« ist vorerst keine passende Antwort, weil Intuitionen nicht als wahrnehmungsähnliche Erscheinungen behandelt werden dürfen, wenn das intuitive gegenüber dem diskursiven Denken korrekt abgegrenzt werden soll (siehe oben, Kap. 3.6). 
Dem internalistischen Befund, wonach Rechtfertigung nur zu verstehen ist, wenn die rechtfertigenden Gründe Gründe für das Subjekt sind, soll hier also gefolgt werden. Daraus ergibt sich für die Intuition, dass die Adaptationsgeschichte zwar möglicherweise einen notwendigen, nicht aber einen hinreichenden Teil einer Erklärung der intuitiven Rechtfertigung bildet. Auf der anderen Seite soll die >Grund-für-das-Subjekt<-Forderung nur im eingeschränkten Sinn gelten, dass deren Erfüllung eine notwendige Bedingung für gelingende Rechtfertigung ist; nicht aber im stärkeren Sinn, dass wir durch die Angabe subjektperspektivischer Gründe die Rechtfertigungserklärung vollständig geben können. Ein solcher Internalismus wäre unplausibel. ${ }^{214}$ Damit ist die Motivation für die nun zu entwickelnde internalistische Erklärung dargelegt, welche die externalistische Adaptationsgeschichte in Bezug auf intuitive Rechtfertigung ergänzen soll.

\subsection{Das Problem der nichtbegrifflichen Rechtfertigung}

Ein internalistischer Ansatz der intuitiven Rechtfertigung wird sich aufgrund des im vorangegangenen Teil der Arbeit erläuterten Intuitionsverständnisses mit einem Problem konfrontiert sehen, das im Folgenden als >Problem der nichtbegrifflichen Rechtfertigung، bezeichnet werden soll.

Zur Erörterung des Problems nichtbegrifflicher Rechtfertigung muss von der oben dargestellten internalistischen Forderung ausgegangen werden, wonach ein Grund nur dann ein Grund für ein Subjekt ist, wenn das Subjekt über diesen Grund verfügt. In einer ersten Annäherung lässt sich sagen, dass dies nur dann möglich ist, wenn der Grund in einem begrifflichen Gehalt besteht, wobei >begrifflich hier bedeutet, dass das Subjekt selber über die entsprechenden Begriffe verfügt, die den Gehalt beschreiben. In dieser Sichtweise wird postuliert, dass sich Rechtfertigungsbeziehungen nicht über den Raum

214 In diesem Punkt soll Thomas Grundmanns Zurückweisung des Internalismus teilweise gefolgt werden:

Wenn die Rechtfertigung allein von unserer Perspektive abhängen würde, dann wäre es vollkommen rätselhaft, warum uns die Rechtfertigung so wichtig erscheint. Angesichts unseres epistemischen Ziels der Wahrheit ist es doch vollkommen gleichgültig, ob es von irgendeinem Standpunkt so aussieht, als ob die Gründe zuverlässig zur Wahrheit führen. Wir sind einzig und allein daran interessiert, dass uns unsere Gründe tatsächlich zur Wahrheit hinführen.

(Grundmann 2008, S. 254).

Aufgrund der obigen Ausführungen möchte ich aber »vollkommen gleichgültig« und »einzig und allein« in Grundmanns Zitat abschwächen, etwa zu »nicht allein entscheidend « und »auch $\ll$. 
des Begrifflichen hinaus erstrecken können. Wäre dies zutreffend, ergäbe sich ein gravierendes Problem für die intuitive Rechtfertigung, da diese als Relation zwischen Intuitionen $_{\mathrm{NP}}$ und intuitiven Überzeugungen bestimmt wurde.

Die enge Verbindung von Rechtfertigung und Propositionalität ist Teil einer philosophischen Auffassung, die ihre Wurzeln bei Kant hat und die in der von Kant und Sellars stark beeinflussten Position John McDowells große Bedeutung erlangt. Im Folgenden soll McDowells Konzeptualismus, ausgehend von seinem vielbeachteten Werk Geist und Welt, genauer betrachtet werden. Dadurch wird sich zeigen, wo die in Bezug auf nichtbegriffliche Rechtfertigung zu lösenden Probleme liegen.

John McDowells zentrales Postulat in Geist und Welt, von dem die für das vorliegende Thema maßgeblichen Argumente abhängen, ist in seinem Ausdruck des >Tribunals der Erfahrung< verdichtet:

Genau das meine ich mit einem »minimalen Empirismus«: der Gedanke, daß die Erfahrung ein Tribunal bilden muß, welches zwischen dem Denken und seiner Verantwortlichkeit gegenüber der Welt vermittelt, und ohne dessen Vermittlung es überhaupt keinen Sinn hätte, es überhaupt als Denken zu bezeichnen. ${ }^{215}$

Die Erfahrung ist McDowell zufolge die Instanz, auf die wir uns zu berufen haben, wenn wir unsere Überzeugungen über die Welt rechtfertigen wollen. Sie vermittelt zwischen unserem Denken »und seiner Verantwortlichkeit gegenüber der Welt«. Aber wie kann die Erfahrung diese Rolle spielen, wenn wir von der Kantischen Annahme der Rezeptivität der Erfahrung ausgehen? Dies ist das zentrale Problem, an dem sich verschiedene Positionen abarbeiten.

Bevor McDowell seine Lösung des Problems präsentiert, weist er andere Lösungsvorschläge zurück. Für die vorliegende Arbeit wichtig ist seine Zurückweisung des epistemischen Fundamentalismus. Dieser vertritt die Auffassung, durch die Erfahrung ein Fundament unserer Erkenntnis dingfest machen zu können, welches den infiniten Regress in Bezug auf Überzeugungen stoppt. Überzeugungen werden gemäß dieser Vorstellung durch Erfahrungen gerechtfertigt, welche ihrerseits nicht weiter gerechtfertigt werden müssen, da sie zugleich, d.h. neben ihrer rechtfertigenden Kraft, gegeben sind. Gegen diese Position wendet McDowell ein, dass sie sich auf eine problematische Rechtfertigungstheorie abstützen muss. Dies lässt sich am Beispiel einer Wahrnehmungsüberzeugung demonstrieren: Warum ist $S$ gerechtfertigt zu sagen, dass vor ihm auf dem Tisch ein roter Apfel liegt? Weil S diesen roten Apfel sieht. Wird nun der Gehalt der Wahrnehmung von S als nichtbegrifflich aufgefasst,

215 McDowell (1998, S. 12). 
so kann höchstens die Theoretikerin von außen sagen, dass S' Überzeugung durch seine Wahrnehmung gerechtfertigt wird, weil diese korrekt funktioniert, indem sie die Lichtreflexion der Apfeloberfläche korrekt aufnimmt und verarbeitet, sodass der Roteindruck entsteht, usw. Hingegen möchten wir gemäß McDowell Gründe haben, die Gründe für S sind. Hiermit wären nichtbegriffliche Gehalte keine Option, da aufgrund einer angenommenen Deckung von Begrifflichkeit und Rechtfertigung >nichtbegrifflich bedeutet, dass S selber nicht über den entsprechenden Grund verfügt. Somit kann es McDowell zufolge keine nichtbegrifflichen Gründe geben. ${ }^{216}$ McDowell insistiert damit in Anlehnung an Sellars, dass ein empirisch Gegebenes, das als solches keine begriffliche Struktur aufweist, keine rechtfertigende Kraft haben kann. ${ }^{217}$

Kommen wir zu McDowells alternativer Antwort auf die Frage, wie die Erfahrung die Rolle eines Tribunals einnehmen kann. McDowells Lösung des Problems besteht darin, bereits die Erfahrung - obwohl auch rezeptiv - als durch und durch begrifflich strukturiert zu verstehen:

Begriffliche Fähigkeiten, deren Beziehungen in den logischen Raum der Gründe sui generis gehören, kommen nicht nur in Urteilen - Ergebnissen aktiver Entscheidungsprozesse seitens der Subjekte - zur Anwendung, sondern bereits in den natürlichen Vorgängen, die durch die Einwirkung der Welt auf die rezeptiven Fähigkeiten geeigneter Subjekte zustande kommen, d.h. auf solche Subjekte, die die entsprechenden Begriffe besitzen. ${ }^{218}$

Da McDowell zufolge jede Erfahrung begrifflichen Gehalt aufweist, befindet sie sich immer bereits im logischen Raum der Gründe und kann somit Überzeugungen rechtfertigen. Dadurch wird die Anforderung erfüllt, wonach sich unsere Überzeugungen vor dem Tribunal der Erfahrung rechtfertigen können müssen.

Wäre McDowells Auffassung des Zusammenhangs zwischen Erfahrung und Rechtfertigung korrekt, bliebe uns die Möglichkeit verwehrt, Intuitionen ${ }_{N P}$ als Gründe zu verstehen, da diese nichtbegriffliche Gehalte aufweisen. Im Folgenden soll diese Auffassung kritisch beleuchtet werden. Um eine zielgerichtete Kritik zu ermöglichen, ist zunächst das konzeptualistische Argument präzise darzulegen. Aufgrund der obigen Ausführungen lässt sich dieses Argument wie folgt verstehen:219

\footnotetext{
216 Vgl. McDowell (1998, S. 31f., 37).

217 Vgl. McDowell (1998, S. 14).

218 McDowell (1998, S. 20).

219 Die folgende Rekonstruktion ist inspiriert durch eine entsprechende Argumentation Bill Brewers für den Konzeptualismus, die im Wesentlichen den dargestellten Gedanken McDowells entspricht (vgl. Brewer 2005). Während Brewer sein Argument auf
} 
(P1) Erfahrungsgehalte liefern Gründe für Überzeugungen, dann und nur dann, wenn sie in passender Beziehung zu Propositionen stehen, die ihrerseits inferentiell aufeinander bezogen sind.

$\left(\mathrm{P}_{2}\right)$ Erfahrungsgehalte stehen in passender Beziehung zu diesen Propositionen, dann und nur dann, wenn sie mit den Gehalten der entsprechenden Propositionen identisch sind.

(P3) Der Gehalt von Propositionen ist begrifflich.

(K) Erfahrungsgehalte liefern Gründe für Überzeugungen, dann und nur dann, wenn deren Gehalt begrifflich ist.

$(\mathrm{P} 1)$ und $\left(\mathrm{P}_{2}\right)$ im obigen Argument beruhen auf einer konzeptualistischen Vorannahme in Bezug auf das Wesen von Begründungen. Wenn wir Begründung allein als inferentielle Beziehung zwischen Propositionen gelten lassen, ist dies eine Einschränkung, für die argumentiert werden müsste, jedoch meines Wissens nie argumentiert wurde. Folgende Passage aus Geist und Welt bringt die oben dargestellte Begrifflichkeitsvoraussetzung klar zum Ausdruck:

Die Beziehungen, jedoch, kraft derer Urteile gerechtfertigt werden, lassen sich nur als Beziehungen im Raum der Begriffe verstehen: Implikations- oder Wahrscheinlichkeitsbeziehungen, die zwischen möglichen Ausübungen der begrifflichen Spontaneität bestehen. ${ }^{220}$

Hier wird auch deutlich, was eine nichtbegriffliche Rechtfertigungstheorie zu leisten hat: Sie muss zeigen, inwiefern die Beziehungen, »kraft derer Urteile gerechtfertigt werden «, zumindest zu einem gewissen Teil auch nichtbegriffliche Beziehungen sein können. Diesen Weg möchte ich im Folgenden mit der Ausarbeitung eines Modells der intuitiven Rechtfertigung beschreiten. Zuvor aber soll das Gelände der Konzeptualismus-Nonkonzeptualismus-Debatte abgesteckt werden, in welchem sich das Modell der intuitiven Rechtfertigung verorten lässt.

Um zu zeigen, inwiefern nichtbegriffliche Gehalte Gründe sein können, müssen zunächst drei Formen des Nonkonzeptualismus voneinander unterschieden werden. Ich werde sie im Folgenden als >naturalistischen Nonkonzeptualismus<, >Nonkonzeptualismus Typ Evans<, sowie >Nonkonzeptualismus der Begründung bezeichnen. Der Nonkonzeptualismus der Begründung ist diejenige Form, die hier vertreten werden soll, da nur er die strikte Bindung von Begründung an Begrifflichkeit lockert.

den Bereich der Sinneswahrnehmung beschränkt, erweitere ich es hier auf Erfahrung im Allgemeinen.

220 McDowell (1998, S. 31). 
Nicht viel mehr ist zum naturalistischen Nonkonzeptualismus zu sagen: Dieser anerkennt keinen vom Raum der Natur unabhängigen Raum der Gründe. Gemeinhin gelten hier Gründe - und generell Normativität - als vollständig reduzierbar auf naturgesetzlich beschreibbare Prozesse. Diese Position bedarf hier keiner weiteren Erörterung, da wir uns im Bereich der internalistischen Rechtfertigung befinden. Ein naturalistischer Nonkonzeptualismus würde entweder die Forderung der internalistischen Rechtfertigung nicht akzeptieren und sich mit der externalistischen Geschichte begnügen, oder er müsste eine Erklärung dafür liefern, wie auf einer tieferliegenden Ebene auch internalistische Gründe naturalisierbar sind.

Der Nonkonzeptualismus Typ Evans hingegen anerkennt einen distinkten Raum der Gründe, wobei er zugleich die Begrifflichkeit der Gründe akzeptiert. Zur Verdeutlichung dieses Nonkonzeptualismus seien zwei Passagen aus Evans' Varieties of Reference angeführt:

It is as well to reserve >belief $\prec$ for the notion of a far more sophisticated cognitive state: one that is connected with (and, in my opinion, defined in terms of) the notion of judgement, and so, also, connected with the notion of reasons. The operations of the informational system are more primitive. ${ }^{221}$

In other words, we arrive at conscious perceptual experience when sensory input is not only connected to behavioural dispositions in the way I have been describing - perhaps in some phylogenetically more ancient part of the brain but also serves as the input to a thinking, concept-applying, and reasoning system; so that the subject's thoughts, plans, and deliberations are also systematically dependent on the informational properties of the input. ${ }^{222}$

Wie in den obigen Passagen deutlich wird, sieht Evans die Konzeptualisierung der informational states als notwendig dafür an, dass diese in eine Begründungsrelation - etwa zu Überzeugungen - treten können. Der nichtbegriffliche empirische Gehalt wird als Input in einen begrifflich operierenden Begründungsapparat aufgefasst. Es verwundert daher nicht, dass McDowell und weitere Konzeptualisten den Nonkonzeptualismus des problematischen Gehalts-Begriffs-Dualismus bezichtigen. ${ }^{223}$ Zwar teilt McDowell mit Evans die Auffassung, dass eine Erfahrung Begrifflichkeit involviert. Jedoch ergibt sich bei Evans die Problematik der Konzeptualisierung, auf die McDowell zu Recht hinweist: ${ }^{24}$ Wird die Begrifflichkeit der Begründung anerkannt, bleibt

\footnotetext{
221 Evans (1982, S. 124).

222 Evans (1982, S. 158).

223 Vgl. Boyle (2016), Lauer (2014, S. 47ff.).

224 Vgl. McDowell (1998, S. 77f.).
} 
rätselhaft, was am Übergang zwischen dem Bereich der informational states und dem Bereich der Gründe geschieht.

Der Nonkonzeptualismus der Begründung knüpft zunächst an McDowells Kritik gegen den Nonkonzeptualismus an. Entscheidend ist, dass McDowells Einwände nur die beiden anderen Varianten des Nonkonzeptualismus treffen. Ein Nonkonzeptualismus der Begründung hingegen anerkennt einerseits das Bestehen eines Raums der Gründe und stimmt dadurch mit McDowells $>$ Tribunal der Erfahrung<-Forderung überein. Andererseits verneint diese Form des Nonkonzeptualismus die Kantische Annahme der Begrifflichkeit der Begründung. Dadurch bleibt Raum für eine Position, welche die internalistische Erfordernis beachtet, wonach Gründe Gründe für ein Subjekt sein müssen, ohne darauf verpflichtet zu sein, dass nur begriffliche Gehalte begründende Kraft haben können.

Wie ist aber nichtbegriffliche Begründung zu denken? Hier soll eingeräumt werden, dass Schließen (inference) - zumindest in der geläufigen Bedeutung dieses Ausdrucks - tatsächlich nur zwischen begrifflichen Gehalten möglich ist. Von nichtpropositionalem Schließen zu sprechen wäre in sich widersprüchlich. Dies bedeutet jedoch nicht, dass Begründungsrelationen ebenfalls nur vollständig begrifflich denkbar sind. Zentral für die Ausarbeitung des Modells intuitiver Rechtfertigung wird sein, den oft stillschweigend vorgenommenen Übergang zwischen Begründung und Schließen zu hinterfragen. Wie wir bei McDowell sehen, ist für Begründung vor allem gefordert, dass der Grund ein Grund für das Subjekt ist und nicht nur aus der Außenperspektive als solcher erkannt werden kann. Der Grund muss also, um als genuiner Grund zu gelten, für das Subjekt verfügbar sein. Warum soll eine solche Verfügbarkeit nur durch eine Beziehung des Schließens gegeben sein? Zumindest besteht die Möglichkeit, dass die Verfügbarkeit von Gründen jenseits inferentieller Beziehungen gegeben sein könnte. Wie eine solche nicht-inferentielle Verfügbarkeit tatsächlich bestehen kann, soll im Folgenden in Bezug auf die Intuition aufgezeigt werden. ${ }^{225}$

\subsection{Grundlagen eines Modells der intuitiven Rechtfertigung}

Im Sinne des Nonkonzeptualismus der Begründung muss gezeigt werden, dass Intuitionen $_{\mathrm{NP}}$ Gründe für Subjekte sein können. Hierzu schlage ich ein Modell

225 Es ist anzunehmen, dass der Nonkonzeptualismus der Begründung auch für andere Erfahrungsgehalte, namentlich für Wahrnehmung, Gültigkeit hat. Hier beschränke ich mich auf die Ausarbeitung in Bezug auf Intuition. 
der intuitiven Rechtfertigung vor. Diesem Modell zufolge ist eine Intuition ${ }_{N P}$ genau dann S' Grund für seine intuitive Überzeugung, wenn Folgendes der Fall ist:

(1) S ist durch ein mentales Modell mit einem die Überzeugung erfüllenden Sachverhalt bekannt.

(2) S kommt durch Typ 1-Denken ausgehend von diesem mentalen Modell zu der Intuition $\mathrm{NP}$.

(3) Der Gehalt der intuitiven Überzeugung ist identisch mit dem Gehalt der Intuition $_{\mathrm{NP}}$.

(4) S ordnet durch mentale Demonstration die Überzeugung als intuitive Überzeugung ein.

Bevor die vier genannten Aspekte genauer erläutert werden, soll auf zwei wichtige Grundlagen für diese Aspekte eingegangen werden. Die erste Grundlage bildet das Konzept der Bekanntschaft, welches eine bestimmte epistemische Relation eines Subjekts zu einem Gegenstand oder Sachverhalt beschreibt. Wie gezeigt werden soll, ist die Bekanntschaftsrelation auch im Fall der intuitiven Rechtfertigung bedeutsam, insofern ein Subjekt aufgrund seiner mentalen Modelle mit bestimmten Sachverhalten bekannt ist. Als zweite Grundlage des Modells der intuitiven Rechtfertigung ist das Konzept des Grundes näher zu betrachten. Hierbei werde ich dafür argumentieren, dass auch Intuitionen $_{N P}$ genuine Gründe für Subjekte sein können. Dies erfordert ein Verständnis von `Grund «, welches es erlaubt, auch nichtpropositionale Gehalte als Gründe einzuordnen. Bekanntschaft und nichtpropositionale Gründe sind die Voraussetzungen dafür, eine auch der internalistischen Anforderung genügende Rechtfertigungsbeziehung zwischen nichtpropositionalen Intuitionen und intuitiven Überzeugungen herstellen zu können.

\subsubsection{Bekanntschaft}

Die Diskussion und Verwendung des Begriffs $>$ Bekanntschaft $<$ (acquaintance) in der zeitgenössischen analytischen Philosophie geht auf die Arbeiten Bertrand Russells zurück. ${ }^{226}$ Russell unterscheidet zwischen Erkenntnis durch Bekanntschaft (knowledge by acquaintance) und Erkenntnis durch Beschreibung (knowledge by description).227 Ohne näher auf die durchaus kontrovers gewordenen Einzelheiten von Russells Erörterung einzugehen, kann hier darauf fokussiert werden, was Russell unter >Bekanntschaft< (in Abgrenzung zu >Beschreibung ) versteht:

226 Vgl. Russell (1910-11; 2001, Kap. 5).

227 Für eine vertiefte Darstellung der beiden Erkenntnisformen siehe Gabriel (2015a, S. 58 f. u.a.). 
I say that I am acquainted with an object when I have a direct cognitive relation to that object, i.e., when I am directly aware of the object itself. When I speak of a cognitive relation here, I do not mean the sort of relation which constitutes judgment, but the sort which constitutes presentation. In fact, I think the relation of subject and object which I call acquaintance is simply the converse of the relation of object and subject which constitutes presentation. That is, to say that $\mathrm{S}$ has acquaintance with $\mathrm{O}$ is essentially the same thing as to say that $\mathrm{O}$ is presented to S. ${ }^{228}$

Russell versteht also unter Bekanntschaft das Sich-Direkt-Bewusstsein (awareness) eines Objekts. Weiter ist der Gegensatz zwischen Urteil (judgment) und Darbietung (presentation) hervorzuheben. Hier wird ersichtlich, inwiefern Bekanntschaft in einem nichtpropositionalen Kontext steht. Die Erkenntnis durch Bekanntschaft ist kein propositionales $>$ Wissen, dass $\mathrm{p}<$, sondern ein Wissen im Sinne des Kennens. Ebenfalls bereits bei Russell angesprochen ist die Feststellung, dass die nichtpropositionale Erkenntnis durch Bekanntschaft immer reichhaltiger als jedes korrespondierende propositionale Wissen ist. So kann keine bestimmbare Menge an propositionalen Urteilen gänzlich das Wissen ausdrücken, welches wir im Sinn des Kennens haben:

Moreover, as we saw earlier in considering the relations of description and acquaintance, we often wish to reach the denotation, and and [sic] are only hindered by lack of acquaintance: in such cases the description is merely the means we employ to get as near as possible to the denotation. ${ }^{229}$

An dieser Stelle lässt sich die herausragende Bedeutung erkennen, die Russell der nichtpropositionalen Erkenntnis durch Bekanntschaft beimisst: In epistemischer Hinsicht ist propositionale Erkenntnis durch Beschreibung oft unvollständig und ein Hilfsmittel, um der Erkenntnis durch Bekanntschaft so nahe wie möglich zu kommen. Mehr noch, Erkenntnis durch Bekanntschaft ist das Fundament, auf welchem unser weiteres Wissen ruht. ${ }^{230}$

Ein weiterer wichtiger Aspekt der Erkenntnis durch Bekanntschaft ist deren Unmittelbarkeit:231

We shall say that we have acquaintance with anything of which we are directly aware, without the intermediary of any process of inference or any knowledge of truths. [...] Thus the sense-data which make up the appearance of my table are

228 Russell (1910-11, S. 108).

229 Russell (1910-11, S. 127).

230 Vgl. Russell (2001, S. 26).

231 Der Aspekt der Unmittelbarkeit kann zu Überhöhungen dieser nichtpropositionalen Erkenntnisform führen, die wiederum starke Kritik auslösen (vgl. Gabriel 2015a, S. 63f.). 
things with which I have acquaintance, things immediately known to me just as they are. ${ }^{232}$

Hier zeigt sich aufgrund der Merkmale der Unmittelbarkeit und Gewissheit ein Bezug der Erkenntnis durch Bekanntschaft zur Intuition. Bekanntschaft versetzt uns in direkte Kenntnis eines Gegenstandes, ohne dass wir diese Kenntnis etwa aus anderen Überzeugungen ableiten. Die nichtpropositionale Erkenntnis durch Bekanntschaft erfolgt Russell zufolge ohne jegliche Vermittlung durch einen Prozess des Schließens.

Welches sind die Gegenstände der Bekanntschaft? In erster Linie nennt Russell hier die Sinnesdaten. In diesem Zusammenhang betont er, dass wir nie mit dem physischen Objekt, sondern nur mit den Sinnesdaten vertraut sind, weil wir nur zu diesen in einer direkter Beziehung stehen. ${ }^{233} \mathrm{Im}$ Weiteren verfügen wir über Wissen durch Bekanntschaft in Bezug auf Erinnerungen, Introspektion, Selbstbewusstsein und Universalien (z.B. Weissheit, Verschiedenheit, Geschwisterlichkeit). ${ }^{234}$ Weitere Bereiche, auf die sich Erkenntnis durch Bekanntschaft beziehen kann, listet Russell zwar nicht auf, bezeichnet die Aufzählung der erwähnten Bereiche jedoch auch nicht als vollständig. ${ }^{235}$ Daher lässt sich nichtpropositionale Erkenntnis durch Bekanntschaft u.a. auch in Bezug auf die folgenden Erkenntniselemente untersuchen: praktisches Wissen im Sinne des knowing how, nichtbegriffliche Gehalte der Erfahrung, die subjektive phänomenale Qualität im Sinn des »wie es ist« etwas zu erleben, den phänomenalen Charakter des Bewusstseins sowie Selbstbewusstsein. ${ }^{236}$ Ein weiterer Bereich, der mit Erkenntnis durch Bekanntschaft in Zusammenhang gebracht werden kann, ist das vorpropositionale Unterscheidungswissen, welches etwa in Bezug auf die Adäquatheit von Definitionen entscheidend ist. ${ }^{237}$

232 Russell (2001, S. 25).

233 In Probleme der Philosophie führt Russell dies am Beispiel eines Tisches aus: Sinnesdaten, die wir empfangen, wenn wir auf den Tisch klopfen, ihn unter verschiedenen Blickwinkeln und Lichteinflüssen betrachten, usw. sind uns bekannt, jedoch nicht der Tisch an sich (vgl. Russell 2001, S. 25f.).

234 Vgl. Russell (2001, S. 26ff.).

235 Zwar widmet Russell in Probleme der Philosophie dem intuitiven Wissen sogar ein eigenes Kapitel, in dem er dieses im klassisch-philosophischen Sinn als Wissen in Bezug auf selbstevidente Wahrheiten einordnet (vgl. Russell 2001, Kap. 11). Aus diesem Kapitel lässt sich aber für die hier vorliegenden Fragen nichts wesentlich Neues gewinnen. Es ist überraschend, dass Russell keinen Bogen vom intuitiven Wissen zurück zum Kapitel über Erkenntnis durch Bekanntschaft schlägt.

236 Vgl. Schildknecht (1999, S. 13).

237 Vgl. Gabriel (2015a, S. 45-55). 
In all den erwähnten Bereichen verfügt das Subjekt über Erkenntnis, die nicht auf eine propositionale Struktur reduziert werden kann, weil sie die von Russell beschriebene Form der Bekanntschaft hat, durch die dem Subjekt ein Gegenstand oder Sachverhalt in unmittelbarer, reichhaltiger und ganzheitlicher Weise präsent ist. Dabei wird das Subjekt durch Bekanntschaft in Kenntnis eines Gehalts gesetzt, ohne dass es über die entsprechenden Begriffe verfügen muss, die diesen Gehalt kanonisch beschreiben. ${ }^{238}$

Aufgrund dieser Eigenschaften der Bekanntschaftsrelation ist es folgerichtig, diese auch auf intuitive Erkenntnis zu beziehen. Hierbei scheinen mir zwei Momente feststellbar, die von der epistemischen Bekanntschaftsrelation geprägt sind. Erstens kennt das Subjekt - im Sinne der Bekanntschaft - den Sachverhalt, der in einem mentalen Modell (oder mehreren mentalen Modellen) für das Subjekt repräsentiert wird. Zweitens ist eine auf die phänomenalen Eigenschaften der Intuition bezogene Bekanntschaft festzustellen: Das Subjekt ist damit bekannt, wie es ist eine intuitive Überzeugung zu haben und auch damit, wie es ist, die aktuelle Überzeugung zu haben. Hier lässt sich an die Ausführungen in Bezug auf die phänomenalen Eigenschaften der Intuition (Kap. 3.1) anknüpfen. Wie dort gezeigt wurde, bringt die Phänomenalität der Intuition ein Sich-Gewahr-Werden seitens des Subjekts in Bezug auf dessen Intuition mit sich. Dies gilt, sobald wir über Intuition »im Vollsinn « sprechen wollen und den Begriff nicht in einem weiteren Sinn, der allgemein Typ 1-Denkprozesse bezeichnet, verwenden. Da es bei der intuitiven Rechtfertigung nun um die Rechtfertigung intuitiver Überzeugungen geht, lassen sich die phänomenalen Eigenschaften der Intuition (Unmittelbarkeit, Gewissheit) präziser auf intuitive Überzeugungen beziehen. Diese phänomenalen Eigenschaften führen dazu, dass dem Subjekt bekannt ist, wie es ist, eine intuitive Überzeugung zu haben.

Die Bekanntschaft des Subjekts mit dem Typ der intuitiven Überzeugung und der einzelnen vorliegenden Überzeugung (via die phänomenalen Eigenschaften der intuitiven Überzeugung) erlaubt ihm folglich eine Einordnung der vorliegenden Überzeugung als intuitive Überzeugung. (Bereits hier sei festgehalten, dass das Subjekt dafür nicht die spezifischen Ausdrücke sintuitiv<, $>$ Überzeugung<, o.ä. zu kennen braucht). Eine solche Einordnung ist durchaus als propositional zu bezeichnen. $>\mathrm{S}$ ordnet $\mathrm{x}$ als $\mathrm{y}$ ein $<$ muss auf die $\mathrm{Zu}-$ schreibung herauslaufen: >S glaubt, dass $\mathrm{x} y$ ist $\mathrm{S}$ benötigt also Begriffe für $\mathrm{x}$ und $\mathrm{y}$. Dies ändert jedoch nichts daran, dass der maßgebliche Teil der Rechtfertigung von $S$ ' intuitiver Überzeugung von der nichtpropositionalen

238 Siehe Fn. 124 zur Definition von begrifflichem bzw. nichtbegrifflichem Gehalt anhand der possession condition. 
Erkenntnisform der Bekanntschaft herrührt, welche einerseits den Gehalt der intuitiven Überzeugung, andererseits die Einordnung dieser Überzeugung als intuitiv stützt.

Ein Bedenken im Hinblick auf die Einbindung von Bekanntschaft in ein Modell intuitiver Rechtfertigung soll bereits an dieser Stelle ausgeräumt werden: Russell definiert Bekanntschaft als das Sich-Direkt-Bewusstsein (awareness) eines Objekts oder einer Tatsache. Ein typisches Merkmal von Intuitionen hingegen ist, dass wir uns der Basis nicht bewusst sind, die zur entsprechenden intuitiven Überzeugung führt. Das Bedenken lässt sich nun mit Bezug auf bereits vorgestellte Beispiele äußern: Ist es nicht ein maßgebliches Kennzeichen der Intuition der Feuerwehrkommandantin Claire oder eines Golfspielers, dass diese jeweils nicht wissen, woher ihre intuitive Überzeugung kommt? Claires Überzeugung »Es ist zu gefährlich für meine Männer, das Gebäude zu betreten« oder die Intuition des Golfspielers »Ich sollte jetzt Eisen 7 wählen « sind dieser Art. Diesem Bedenken zufolge wäre also in solchen Beispielen keine Bekanntschaft mit dem Sachverhalt festzustellen, auf dem die Intuition beruht, falls wir Russells Erfordernis des Sich-direkt-Bewusstseins ernst nehmen. Somit wäre dem ersten der oben beschriebenen Einflüsse der Bekanntschaft in das Modell der intuitiven Rechtfertigung bereits der Boden entzogen: der Bekanntschaft mit dem Sachverhalt, der durch das mentale Modell repräsentiert wird. Demnach wäre es nicht statthaft, die Bekanntschaftsbeziehung in Bezug auf die Gehalte der Intuition in Anschlag zu bringen.

Dem vorgebrachten Einwand gegen die Einführung der Bekanntschaftsrelation in den Bereich der intuitiven Erkenntnis lässt sich auf zwei Weisen begegnen. Erstens muss das Sich-Direkt-Bewusstsein eines Sachverhaltes nicht notwendigerweise beinhalten, dass ein Subjekt alle Aspekte dieses Sachverhaltes auflisten kann. Dies sehen wir am Beispiel des Golfspielers: In einem gut nachvollziehbaren Sinn ist sich dieser der gesamten Situation auf dem Golfplatz bewusst. Er steht dort und nimmt alle relevanten Aspekte wahr, die den Sachverhalt konstituieren (was angenommen werden soll, wenn wir seine Intuition von einem mentalen Modell ausgehend erklären, das diesen Sachverhalt abbildet). In einem ähnlichen Sinn lässt sich auch sagen, dass jemand, der ein aus vielen fein voneinander unterschiedenen Farbtönen aufgebautes abstraktes Gemälde als Ganzes erfasst, sich dieses Gemäldes direkt bewusst ist, ohne dass er die einzelnen Farbtöne auflisten können muss. Das für Wahrnehmung wie auch für Intuition charakteristische Merkmal des schlagartigen Erfassens besagt ja gerade, dass ein Subjekt sich der Sache direkt bewusst ist, und zwar schlagartig. 
Die zweite Replik lautet: Sogar wenn wir einer Kritikerin zugestehen, dass der Golfspieler sich des Sachverhalts nicht vollständig direkt bewusst ist, impliziert dies nicht, dass wir auf die Einführung der Bekanntschaftsrelation in den Bereich der intuitiven Erkenntnis verzichten müssen. Diese Replik stützt sich darauf, dass wir philosophische Konzepte übernehmen können, ohne alle Einzelheiten ihrer ursprünglichen Formulierung mitnehmen zu müssen. Bereits erwähnt wurde die mögliche Ausweitung der Bekanntschaftsrelation, beispielsweise auf Sachverhalte und auf phänomenale Erkenntnis. Solche Ausweitungen legitimieren sich jeweils dadurch, dass der Urheber des Konzepts dieses schlicht nur für bestimmte Problemstellungen verwendet hatte. So war es für Russells Problemstellung nicht von Bedeutung über Ebenen nachzudenken, auf denen dem Subjekt etwas nicht direkt bewusst ist, da es ihm vorrangig um die Abgrenzung der beiden Erkenntnisformen (knowledge by acquaintance und knowledge by description) ging. Diese konnte Russell einfacher dadurch vornehmen, dass die beiden Formen unter der konstant gehaltenen Variable $>$ Bewusstheit< miteinander verglichen wurden. In diesem Sinn wurde \Sich-Direkt-Bewusstsein $<$ mit `Sich-Bewusstsein-dass< verglichen. Das Sich-Direkt-Bewusstsein ist aber keine notwendige Bedingung für Bekanntschaft, wie sich anhand von Beispielen plausibilisieren lässt: So ist es durchaus sinnvoll, von Bekanntschaft mit Toms Gesicht zu sprechen, auch wenn ich mir nicht jeder Einzelheit von Toms Gesicht im gegebenen Moment direkt bewusst bin. Bekanntschaft soll daher im Folgenden in einem Sinn verstanden werden, der Sich-Direkt-Bewusstsein nicht miteinschließt.

Die Ausführungen zur Bekanntschaft zusammenfassend lässt sich festhalten: Die Bekanntschaftsrelation ist ein zentrales Merkmal der epistemischen Situation, in der sich ein Subjekt im Hinblick auf seine Intuitionen befindet. Der Einbau der Bekanntschaftsrelation in das Modell intuitiver Rechtfertigung wird dadurch ermöglicht, dass es durchaus sinnvoll ist, unter Bekanntschaft auch (entgegen des ursprünglichen Russell-Verständnisses) Gehalte zu subsumieren, die dem Subjekt nicht bewusst zugänglich sind. ${ }^{239}$ Diese Auffassung

239 In einer weiteren Hinsicht hebt sich das hier vertretene Verständnis von Bekanntschaft von einem verbreiteten Verständnis ab: Oft wird angenommen, es könne einem nur etwas bekannt sein, das existiert. In dieser Hinsicht sei >bekannt sein mit< vergleichbar mit beispielsweise >etwas werfen< (vgl. Hasan/Fumerton 2017). Ich halte dieses Verständnis für unplausibel. Durchaus ist es sinnvoll zu sagen, dass Toms Gesicht S bekannt ist, auch wenn sich später herausstellen sollte, dass Tom eine illusionäre Figur war, die S sich aufgrund einer psychischen Störung einbildete. Andernfalls müsste man beispielsweise auch für `Sehen festhalten, dies garantiere die Existenz des Gesehenen. In einem primären Sinn ist dies sicher nicht der Fall. 
kann wesentlich zu einer Klärung beitragen, inwiefern die nichtbegrifflichen Gehalte, welche via mentale Modelle Grundlage der Intuition sind, Gründe für ein Subjekt sein können. Wie ersichtlich wurde, können die Intuitionen ${ }_{N P}$ isoliert noch nicht als Gründe gelten, doch eingebettet in die erwähnten Bekanntschaftsrelationen werden sie solche Gründe. Wie dies genauer vonstattengeht, wird im Folgenden auszuarbeiten sein.

\subsubsection{Nichtpropositionale Gründe}

Neben der Bekanntschaft ist ein geeignetes Gründe-Verständnis die zweite Säule des Modells der intuitiven Rechtfertigung. Um sich diesem Verständnis anzunähern, sei zunächst an die Cartesische Intuition »ich denke, ich existiere« erinnert: Descartes weist darauf hin, dass die Existenz unmittelbar aus dem Denken einsichtig wird. Dadurch, dass ich denke, kann ich unmittelbar erkennen, dass ich existiere. Das deduktive Schließen müsste im Gegensatz dazu über mindestens eine weitere Prämisse laufen. ${ }^{240}$ Es ist instruktiv, dieses klassische Verständnis der intuitiven Erkenntnis als Prüfstein für ein geeignetes Gründe-Verständnis zu nehmen. Was genau, so lautet die zentrale Frage, fungiert in Descartes' »ich denke, ich existiere « als Grund wofür? Dieser Frage soll im Folgenden nachgegangen und dabei ein Verständnis von >Grund < gestützt werden, welches zulassen wird, Intuitionen ${ }_{\mathrm{NP}}$ als Gründe zu bezeichnen.

Descartes' Gegenüberstellung von Intuition und Deduktion legt zunächst nahe, intuitive Erkenntnis mit unmittelbaren Schlüssen gleichzusetzen, also mit Schlüssen, die aus einer einzelnen Prämisse hervorgehen. ${ }^{241}$ Dies könnte zu der Folgeannahme führen, dass diese einzelne Ausgangsprämisse, also z.B. $>$ ich denke`, der intuitive Grund ist, um den es hier geht. Ein solches Verständnis von intuitiven Gründen würde uns aber auf eine falsche Fährte führen. Dies lässt sich daran erkennen, dass die Prämisse eines unmittelbaren Schlusses so komplex sein kann, dass dieser Schluss nicht mehr als intuitiv gelten darf: »Descartes denkt. Also: Es ist nicht der Fall, dass nicht der Fall ist, dass nicht der Fall ist, dass Descartes nicht denkt.« Es mag Menschen geben, die intuitiv von der Richtigkeit dieses Schlusses überzeugt sind, aber bei den meisten dürfte dies nicht der Fall sein, sondern sie müssen in Schritten herleiten, dass die Konklusion aus der Prämisse folgt. Dadurch zeigt sich, dass ein unmittelbarer Schluss nicht einer intuitiven Erkenntnis entspricht und somit nicht einfach die Ausgangsprämisse eines unmittelbaren Schlusses mit dem Grund

240 Z.B.: »Ich denke, wenn etwas denkt, dann existiert es, also: ich existiere«.

241 Vgl. Kant, AA IX, §42; sowie dazu Gabriel (2015a, S. 63f.). 
identifiziert werden darf, um den es im Fall der intuitiven Rechtfertigung geht. ${ }^{242}$ Den relevanten Grund einer intuitiven Rechtfertigungsbeziehung erhalten wir vielmehr, wenn wir das entscheidende Merkmal der Cartesischen intuitiven Erkenntnis beachten. Dieses ist nicht eine Herleitung aus nur einer Prämisse, sondern die Ganzheitlichkeit, mit der die Prämisse bzw. Prämissen und die Konklusion in den gemeinsamen Blick genommen werden. Im Sinne der Wortverwandtschaft zwischen intueri und >Erblicken« wird hier nicht schrittweise deduziert, sondern auf einen Blick die Gültigkeit erkannt. ${ }^{243}$ So wird der Zusammenhang zwischen >ich denke< und >ich existiere auf einen Blick erfasst, ohne eine schrittweise Ableitung vorzunehmen.

Bei Descartes lässt sich die Ganzheitlichkeit als zentrales Merkmal der intuitiven Erkenntnis auf mehreren Ebenen feststellen: Einerseits ist bei einer deduktiven Schlusskette jeder kleinste Schritt eine intuitive Erkenntnis, da hier keine weiteren deduktiven Schritte vermitteln können. Intuitive Erkenntnisse bilden somit die Grundlage aller deduktiven Schlüsse. Andererseits lassen sich Descartes zufolge auch längere deduktive Schlussketten in intuitive Erkenntnisse transformieren; dadurch, dass in diesen Fällen das Folgen der Konklusion aus den Prämissen unmittelbar erkannt wird. ${ }^{244}$ So ließe sich im obigen Beispiel eine in Logik versierte Person denken, die dieses Folgen intuitiv erkennt. Gemeinsames Merkmal ist in diesen Fällen - sei es bei den kleinsten deduktiven Schritten oder bei einer schlagartig erfassten langen deduktiven Kette - die Ganzheitlichkeit der intuitiven Erkenntnis.

Was bedeutet nun dieses Verständnis der intuitiven Erkenntnis als ganzheitliches Erfassen für die Gründe-Relation, die das noch zu entwickelnde Modell intuitiver Rechtfertigung beinhalten soll? Angewandt auf das Cartesische Beispiel möchte ich zur Beantwortung dieser Frage folgende These vertreten: Nicht der unmittelbare Übergang von >ich denke< zu sich existiere das eigentlich Intuitive dieser Erkenntnis aus; vielmehr kommt die Intuition bei Descartes erst dadurch ins Spiel, dass er eine Überzeugung in Bezug auf das Folgen von >ich existiere $<$ aus >ich denke $<$ hat - und diese ist eine intuitive

242 >Unmittelbarkeit hat im Kantischen Verständnis des unmittelbaren Schlusses nicht dieselbe Bedeutung wie >Unmittelbarkeitく als phänomenales Merkmal der Intuition. >Unmittelbar bezeichnet bei Kant eine besondere Art des Schlusses, wie oben beschrieben, nicht eine besondere Art des Schließens im phänomenalen Sinn. Mit der hier festgehaltenen Unterscheidung zwischen unmittelbaren und intuitiven Schlüssen soll also keineswegs wieder in Abrede gestellt werden, dass Unmittelbarkeit ein phänomenales Merkmal intuitiver Überzeugungen ist.

243 Der Begriff der Gleichzeitigkeit wäre hingegen verfänglich, da er dazu verleitet, das hier besprochene Verhältnis als ein rein zeitliches aufzufassen. Es besteht vielmehr ein qualitativer Unterschied; intuitives Schließen ist mehr als schnelles deduktives Schließen.

244 Vgl. Descartes, AT X, 387 f. 
Überzeugung. ${ }^{245}$ Dadurch können wir die Cartesische Intuition und Beispiele wie dasjenige der Feuerwehrkommandantin in Bezug auf die Rechtfertigung analog verstehen: Stets geht es um die Rechtfertigung einer bestimmten Art von Überzeugung, nämlich eines solchen Überzeugungstyps, der durch seine zugleich auftretenden phänomenalen Merkmale Unmittelbarkeit und Gewissheit bestimmt ist. Bei Descartes besteht die intuitive Überzeugung darin, dass >ich existiere< aus >ich denke< folgt, denn er ist sich dieses Folgens gewiss und es erscheint ihm unmittelbar. Die Feuerwehrkommandantin ist sich nicht eines Folgens, sondern direkt dessen gewiss, dass es zu gefährlich ist, das brennende Gebäude zu betreten. Mit Bezug auf die einleitende Frage (was genau fungiert bei intuitiver Erkenntnis als Grund wofür?) lässt sich durch diese Gemeinsamkeit festhalten, dass bei der intuitiven Erkenntnis stets ein noch zu bestimmendes $\mathrm{X}$ als Grund für eine intuitive Überzeugung fungiert, wobei $\mathrm{X}$ nicht einfach mit einer bestimmten Aussage gleichgesetzt werden kann.

Welches ist dieses X etwa im Beispiel der Feuerwehrkommandantin Claire? Dies ist in jedem Fall keine einzelne Überzeugung. Vielmehr ist eine Vielzahl von Wissenselementen und deren Relation maßgebend dafür, dass Claire zu ihrer intuitiven Überzeugung kommen kann. Diese müssen also allesamt Bestandteile des Grundes der intuitiven Überzeugung sein. Mentale Modelle der Gesamtsituation, auch bisherige Erfahrung und Wissen beinhaltend, werden hier entscheidend. Doch ist noch unklar, wie solche mentalen Modelle die internalistische Gründe-Erfordernis erfüllen können: Wie kann das, woraus sich Claires intuitive Überzeugung herleitet, ein genuiner Grund für Claire selber sein, wenn doch das entscheidende Merkmal der intuitiven Überzeugung ist, dass sie sich gerade nicht aus einer anderen Überzeugung oder Wahrnehmung deduzieren lässt? Das Modell der intuitiven Rechtfertigung wird eine Antwort auf diese Frage bieten.

245 Ein Einwand gegen die hier vertretene These könnte sein: Es gibt doch durchaus ein verbreitetes Verständnis von >Intuition<, welches >ich denkeくals intuitiven Grund für >ich existiere nimmt und die Intuition nicht erst so ins Spiel kommen lässt, wie es die These zum Ausdruck bringt.

Dieser Befund trifft wohl zu, jedoch dient er nicht als Einwand zu der hier vorgebrachten These. Dies wird ersichtlich, wenn wir den Fall Descartes' mit einem Fall kontrastieren, in dem ein Subjekt lediglich die intuitive Überzeugung hat: >ich existiereく. Möglicherweise kann auch dies als intuitive Überzeugung, also auf mentalen Modellen basierend, gesehen werden. Doch sagt uns Descartes ja nicht bloß, er sei überzeugt, dass er existiere und verweist als Begründung auf seine Intuition. Dies wäre für eine philosophische Abhandlung kaum zufriedenstellend. Vielmehr appelliert Descartes an unsere Intuition in Bezug auf das unmittelbare Folgen von >ich existiere erst einen interessanten Begründungszusammenhang herstellt. 
Als letzte Präliminarie muss auf einen skeptischen Einwand gegen die erkenntnistheoretische Relevanz der Intuition eingegangen werden, der, wenn zutreffend, die soeben begonnenen Bemühungen, eine spezifische Gründe-Relation für die intuitive Erkenntnis ausmachen zu wollen, zunichtemachen würde. Dieser Einwand ergibt sich im Anschluss an die folgende Überlegung Gottfried Gabriels:

Das Faktum unmittelbarer intellektueller Einsichten wird niemand als Indiz
einer höheren Erkenntnisfähigkeit, etwa der Intuition im Sinne intellektueller
Anschauung anführen wollen. Vielmehr wird man sagen, dass wir in unserem
Denken implizit Regeln folgen, die uns vielfach gar nicht bewusst sind, so wie
wir auch grammatischen Regeln folgen, ohne diese explizit auf den Begriff ge-
bracht zu haben. Anders gesagt: Von diesen Regeln haben wir eine klare, aber
keine deutliche Erkenntnis. Unmittelbarkeit besagt hier nur, dass es sich um
die Abkürzung eines komplizierten Verfahrens handelt. Der Terminus >intuitiv
charakterisiert dabei lediglich den Weg, nicht aber eine besondere Art der
Erkenntnis. ${ }^{246}$

Gabriel unterscheidet hier zwischen Erkenntnispsychologie bzw. -heuristik und Erkenntnistheorie. Die beschriebene Funktion der Intuition, Schlüsse ganzheitlich zu präsentieren, sodass die Folgerung »auf einen Blick« geschehen kann, ordnet er dem ersten Bereich zu und spricht ihr epistemologische Relevanz ab. Wenn intuitive Erkenntnis nur eine psychologische und keine epistemologische Kategorie wäre, könnte sie lediglich als »Abkürzung eines komplizierten Verfahrens« verstanden werden. Dies würde für die Gründe-Relation bedeuten, dass die Gründe genau dieselben blieben, wie wenn das Verfahren nicht abgekürzt, also deduktiv, durchgeführt worden wäre.

Als Reaktion auf Gabriels Skepsis lässt sich die epistemologische Relevanz der intuitiven Erkenntnis verteidigen. Erstens ist diese Relevanz durch die von Descartes festgestellte Fundierung aller Schlussfolgerungen in kleinsten intuitiv erkennbaren Schritten des Schließens zu sehen. Hier zeigt sich, dass ohne Intuition deduktives Schließen nicht möglich wäre - eine epistemologisch zweifellos bedeutsame Entdeckung, die sich in den größeren Rahmen der Fundierung der propositionalen Erkenntnisebene durch die nichtpropositionale Ebene einfügt. Zweitens ist die Intuition insofern epistemologisch bedeutsam, als sie nicht lediglich eine graduelle Steigerung einer Fähigkeit ist, sondern vielmehr eine neue Erkenntnisqualität ins Spiel bringt. Drittens, und dies ist entscheidend, kann Intuition im Sinne eines

246 Vgl. Gabriel (2015a, S. 64). 
ganzheitlichen Erfassens Erkenntnisse hervorbringen, die auf deduktivem Weg nicht zustande gekommen wären. Als Beispiel sei an Goethes Suche nach der Urpflanze erinnert: Durch das Betrachten der Teile (vieler Wachstumsstadien der Pflanzen) und der Übergänge zwischen den Teilen kam Goethe zur Wesenseinsicht in die >Urpflanze<, aus der er in späteren Schritten noch nicht beobachtete Einzelpflanzen und -stadien ableiten konnte. Dass in einem solchen Fall dieselben Erkenntnisse möglicherweise auch deduktiv abgeleitet werden können, sollte nicht zu hoch gewichtet werden. Falls diese Erkenntnisse in der Art Goethes gefunden werden können, ist der Nachweis einer eigenständigen, epistemologisch bedeutsamen Erkenntnisart bereits erbracht.

Obwohl die epistemologische Relevanz der Intuition bestehen bleibt, muss eine Einschränkung hinsichtlich ihres epistemischen Status festgehalten werden, die mit der bereits erteilten Absage an die Infallibilität der Intuition verwandt ist: In der Erkenntnis geht es mit rechten Dingen zu. Auch die Intuition kann nur mit dem Material arbeiten, das dem Erkenntnissubjekt zur Verfügung steht. So ist Goethes Herleitung der 〉Urpflanze<nichts Mysteriöses. An der Basis stehen Erfahrungen mit spezifischen Gehalten (die Beobachtungen einzelner Pflanzen), aus denen Goethe auf intuitive Weise neue Erkenntnisse gewinnt. Dies muss nicht zur Annahme führen, die Intuition sei eine Erkenntnisart, welche die menschlichen Fähigkeiten der Wahrnehmung und Kognition in irgendeiner Weise transzendiere. Vielmehr sind nichtbegriffliche Erfahrungsgehalte entscheidend, aus denen intuitive Erkenntnisse abgeleitet wurden. Auch im Rahmen der menschlichen Erkenntnismöglichkeiten behält die Intuition dadurch ihre epistemische Bedeutsamkeit.

Die Untersuchung der Gründe-Relation in Bezug auf intuitive Überzeugungen hat bisher somit Folgendes erwiesen: Diese Gründe-Relation kann immer als Beziehung zwischen einem noch näher zu bestimmenden $\mathrm{X}$ und intuitiven Überzeugungen verstanden werden. Dies gilt für die klassischen Intuitionen Descartes' ebenso wie für die in der zeitgenössischen Psychologie betrachteten Intuitionen. Das noch näher zu bestimmende X muss einen engen Zusammenhang zu den mentalen Modellen aufweisen, durch welche dem Subjekt der relevante Sachverhalt, auf den sich die Intuition bezieht, bekannt ist. Die damit angedeutete Suche nach einer spezifischen Gründe-Relation für intuitive Überzeugungen wurde schließlich gegen ein Bedenken verteidigt, welches die Intuition als rein psychologisch zu verstehende Abkürzung des Denkens einordnet. Das nun näher zu erläuternde Modell der intuitiven Rechtfertigung muss die Suche nach der Gründe-Relation aufnehmen und das oben erwähnte $\mathrm{X}$ näher bestimmen. Dabei darf nie vergessen werden, dass der Grund ein genuiner Grund für das erkennende Subjekt sein, also die internalistische Erfordernis erfüllen muss. 


\subsubsection{Das Modell der intuitiven Rechtfertigung}

Dies ist die Ausgangslage: Das Problem der intuitiven Rechtfertigung wurde auf die Frage fokussiert, inwiefern intuitive Überzeugungen (propositional, begrifflich) durch Intuitionen ${ }_{\mathrm{NP}}$ gerechtfertigt sein können. Dabei wurden wir mit dem Problem konfrontiert, wie nichtbegriffliche Gehalte rechtfertigende Kraft haben können. Im Gegensatz zu McDowell, der diese Möglichkeit bestreitet, aber auch zu Evans, der eine vorherige Konzeptualisierung nichtbegrifflicher Gehalte als notwendig erachtet, wurde hier die Begrifflichkeitsthese der Begründung abgelehnt: Begründung, so der Gedanke, kann auch aus internalistischer Perspektive von nichtbegrifflichen Gehalten ausgehen. Um diesen Gedanken auf intuitive Rechtfertigung anwenden zu können, wurde zunächst an Russells Konzept der Bekanntschaft erinnert. Als weitere Grundlage der internalistisch verstandenen intuitiven Rechtfertigung wurde sodann näher bestimmt, um welche Gründe-Relation es geht. Der durch die Ausführungen zu McDowell und Evans geschaffene Raum soll nun mit dem Modell der intuitiven Rechtfertigung ausgefüllt werden. $\mathrm{Zu}$ diesem Zweck werde ich die vier bereits erwähnten Elemente des Modells in unterschiedlicher Ausführlichkeit erläutern.

(1) S ist durch ein mentales Modell mit einem die Überzeugung erfüllenden Sachverhalt bekannt.

Erinnern wir uns an das Beispiel der Feuerwehrkommandantin Claire: Stellvertretend für eine Vielzahl ähnlicher Situationen wurde hier beschrieben, inwiefern Claire aufgrund ihres mentalen Modells der Situation an der Brandstätte zu einer Intuition $\mathrm{NP}_{\mathrm{N}}$ oder zu einer intuitiven Überzeugung kommt. Hierbei ermöglicht die Reichhaltigkeit des mentalen Modells, bereits verfügbare aber noch nicht genutzte Information durch bestimmte kognitive Vorgänge präsent werden zu lassen. Aufgrund der obigen Ausführungen zur Erkenntnis durch Bekanntschaft lässt sich feststellen, dass Claire dank ihres mentalen Modells (oder mehrerer Modelle) in einer Bekanntschaftsrelation zum relevanten Sachverhalt stehen muss. ${ }^{247}$ Der Sachverhalt ist insofern relevant,

247 Der Begriff >Sachverhalt< impliziert ein Verhalten von Sachen zueinander. Siehe hierzu Wittgenstein im Tractatus:

2.0272 Die Konfiguration der Gegenstände bildet den Sachverhalt.

2.03 Im Sachverhalt hängen die Gegenstände ineinander, wie die Glieder einer Kette.

2.031 Im Sachverhalt verhalten sich die Gegenstände in bestimmter Art und Weise zueinander.

2.032 Die Art und Weise, wie die Gegenstände im Sachverhalt zusammenhängen, ist die Struktur des Sachverhalts.

(Wittgenstein, T)

Diese Eigenschaften des Sachverhaltes erlauben dessen isomorphe Repräsentation in mentalen Modellen. 
als er die intuitive Überzeugung Claires erfüllt. >Erfüllen < lässt sich zunächst gut aus der Außenperspektive verstehen: Falls wir den gesamten Sachverhalt kennen würden, könnten wir daraus auch erkennen, dass die Überzeugung wahr ist. Oder einfacher: Die intuitive Überzeugung Claires ist deshalb wahr, weil ein Sachverhalt besteht, der sie wahr macht. Nehmen wir zum besseren Verständnis einen Kontrastfall an: Würde der Sachverhalt in all seiner Komplexität letztlich eine ungefährliche Situation für die Feuerwehrleute implizieren, würde dieser Sachverhalt Claires Überzeugung nicht erfüllen, womit die Überzeugung falsch wäre. Wenn nun der Sachverhalt entscheidend dafür ist, dass Claires Überzeugung wahr ist, muss er Claire in geeigneter Weise zugänglich sein. Nur wenn die entscheidende Rolle, die der Sachverhalt spielt, auch für Claire selbst zum Tragen kommt, hat Claire einen Grund für ihre intuitive Überzeugung. Die Zugänglichkeit des Sachverhalts für Claire ist also eine notwendige Bedingung dafür, dass ihre intuitive Überzeugung eine begründete Überzeugung ist.

Die Zugänglichkeit des Sachverhaltes ist für Claire durch ihre Bekanntschaft mit dem Sachverhalt gegeben: Claire kennt die Situation an der Brandstätte. Das Verb >kennen< ist hier insofern treffend, als die Reichhaltigkeit des Sachverhalts dadurch zum Ausdruck kommt: Windrichtungen, Brandherde, Temperaturen, die Anordnungen der Gebäude im Raum, Materialbeschaffenheit und vieles mehr tritt sowohl in Bezug auf die aktuelle Situation an der Brandstätte als auch in Bezug auf Erinnerungen und Kenntnisse Claires in Erscheinung. Wie bereits plausibilisiert wurde (Kap. 3.4), wird einem Subjekt diese Reichhaltigkeit aufzunehmen zumindest zu einem großen Teil dadurch ermöglicht, dass es Erfahrungen und Kenntnisse in mentalen Modellen repräsentieren kann. Da diese aufgrund ihrer Ikonizität in einer isomorphen Beziehung zu den Sachverhalten stehen, können sie Informationen enthalten, die reichhaltiger sind als jede propositionale Beschreibung, insbesondere reichhaltiger als eine (möglicherweise sehr lange) Liste von Überzeugungen, über die das Subjekt verfügt.

Inwiefern solche mentale Modelle Gründe verfügbar machen, lässt sich durch ein einfaches Beispiel illustrieren: Ein Subjekt, welches sich in einem Gebäude befindet, weiß Teile dieses Gebäudes im dreidimensionalen Raum vor, hinter, ober- und unterhalb von sich, es spürt den Boden unter den Füssen, hört Geräusche aus der unteren oder oberen Etage, usw. Die Mehrzahl dieser Repräsentationen spielt sich auf der Typ 1-Ebene ab, d.h. sie sind nicht explizit und haben keinen begrifflichen Gehalt; $S$ steht nicht in einer propositionalen Einstellung zu ihnen. Dennoch ist die Gebäudewahrnehmung als mentale Repräsentation für $\mathrm{S}$ in sich strukturiert; $\mathrm{S}$ hat also ein mentales Modell des Gebäudes. Dies lässt sich beispielsweise dadurch erkennen, dass sich $\mathrm{S}$ aufgrund seines räumlich-mentalen Modells im Gebäude fortbewegen oder 
Überzeugungen aufgrund des mentalen Modells bilden kann (z.B. die Überzeugung, dass eine obere Etage vorhanden ist). In diesem Sinn ist es korrekt zu sagen, dass das mentale Modell des Gebäudes nichtbegriffliche Gründe für $S$ ' Überzeugung liefert. Mentale Modelle werden hierbei insbesondere durch ihre Ikonizität zur Quelle von Gründen. Die Ikonizität impliziert nämlich, dass in mentalen Modellen reichhaltige Beziehungen enthalten sind, die sabgelesen< werden können. ${ }^{248}$

Was bewirkt, dass der Inhalt des mentalen Modells, verstanden als strukturiertes Ensemble nichtbegrifflicher Gehalte, in eine rechtfertigende Relation zu S tritt und Gründe für S liefert? Hier ist die Bekanntschaft entscheidend. Die Feuerwehrfrau kennt sich mit dem Sachverhalt aus. Nehmen wir als Kontrast dazu an, jemand ohne jegliche Feuerwehrerfahrung würde sich in derselben Situation befinden: Viele nichtbegriffliche Wahrnehmungsgehalte wären wohl wie bei der Feuerwehrkommandantin gegeben, jedoch würden sich diese durch die mangelnde Erfahrung des Laien nicht oder nur bruchstückhaft zu einem mentalen Modell fügen. Durch diese Kontrastierung erhalten wir einen Beleg dafür, dass die Bekanntschaft mit dem Sachverhalt, vermittelt über ein mentales Modell, wesentlich für eine internalistische Rechtfertigungsrelation ist. Im Kontrastbeispiel macht der Laie größtenteils dieselben Typ 1-Wahrnehmungen, ohne dass sich diese zu einem adäquaten mentalen Modell zusammenfügen, was folglich verhindert, dass der Sachverhalt für $\mathrm{S}$ verfügbar ist. Da der jeweilige Sacherhalt entscheidend für die Wahrheit der fraglichen intuitiven Überzeugung ist, muss eine solche Verfügbarkeit und damit die Repräsentation des Sachverhaltes in einem geeigneten mentalen Modell für S gegeben sein.

Eine Kritik an der geschilderten Position könnte darauf insistieren, dass sich der Unterschied zwischen den beiden Situationen allein aus der externalistischen Perspektive hinreichend aufzeigen lässt: Das eine Subjekt verfügt über das entsprechende mentale Modell, das andere eben nicht. Aber was heisst >verfügen über` anderes, als das Modell eben zur Verfügung zu haben und es somit als Grund einspannen zu können? Durch die Bekanntschaftsrelation, die das mentale Modell zwischen Subjekt und Sachverhalt konstituiert, unterscheidet sich das mentale Modell von anderen bewussten und unbewussten Repräsentationen, die Ausschnitte der Wirklichkeit als

248 Der hier entwickelte Gedanke hat Ähnlichkeiten mit der Theorie der Szenario-Gehalte des Nonkonzeptualisten Christopher Peacocke (vgl. Peacocke 1992). Peacockes Theorie wurde aber von konzeptualistischer Seite dahingehend kritisiert, dass sie trotz aller Spezifikation der nichtbegrifflichen Gehalte nicht erwiesen hat, inwiefern diese Gehalte Gründe für das Subjekt sein können (vgl. McDowell 1998, S. 191-199). Dieser Nachweis soll im vorliegenden Vorschlag erbracht werden. 
Gehalt haben. Wir können daher sinnvoll sagen: »Ein Subjekt verfügt über ein mentales Modell«, nicht aber z.B.: »ein Subjekt verfügt über eine Wahrnehmung«, denn es nimmt einfach wahr.

Mit den Ausführungen zum ersten Element des Modells intuitiver Rechtfertigung sollte plausibel geworden sein, dass die Bekanntschaft mit dem Sachverhalt eine notwendige Bedingung dafür ist, dass S' auf diesen Sachverhalt bezogene intuitive Überzeugung für $S$ begründet sein kann. Außerdem konnte gezeigt werden, dass mentale Modelle die Bekanntschaft mit dem Sachverhalt herstellen können. Noch nicht klar ist jedoch, wie die Rechtfertigungsbeziehung genauer aufgebaut ist. Eine nichtpropositionale Bekanntschaftsrelation allein kann noch keinen Grund für eine Überzeugung abgeben. Auch ist noch nicht klar, wie genau die Intuition hier ins Spiel kommt, d.h. welche Rolle es für die Rechtfertigung spielt, dass es sich um eine intuitive (und nicht um eine beliebige andere) Überzeugung handelt. Diese Lücken sollen mit den Ausführungen zu den weiteren Elementen des Modells geschlossen werden.

(2) S kommt durch Typ 1-Denken ausgehend von diesem mentalen Modell zu der Intuition $\mathrm{NP}$.

Durch die Bekanntschaft mit einem Sachverhalt via mentales Modell wird also die Möglichkeit geschaffen, dass der Sachverhalt Gründe für S liefert. Doch was ist notwendig dafür, dass dies tatsächlich erfolgt? Hier muss eine Folgerungsbeziehung dingfest gemacht werden können, die der internalistischen Anforderung genügt: Wenn $S$ etwas als Grund für seine Überzeugung nimmt, impliziert dies, dass $S$ in einer bestimmten Form von diesem Grund auf den Gehalt seiner Überzeugung schließt. Mit dem zweiten Element des Modells sind wir aber noch nicht bei der Folgerung, denn der philosophischen Tradition des Folgerungsbegriffs sei hier - im Gegensatz zu ungenaueren psychologischen Redeweisen - zugestanden, dass genuine Folgerungsbeziehungen nicht einfach im Bereich des Typ 1-Denkens verortet werden können. Dies ist auch der Grund dafür, warum in (2) die Ausdrucksweise »kommt auf« gewählt wurde und nicht etwa »schließt«. Die Prozesse, die unbewusst, schnell und nichtpropositional ablaufen, sind zunächst unkontrolliert, und dies mindestens in doppelter Hinsicht: Sie entziehen sich der bewussten Kontrolle des Erkenntnissubjekts und sie entziehen sich jeglicher formalen Festlegung, etwa der Möglichkeit, sie in Worte zu fassen und damit intersubjektiv nachprüfbar zu machen. Dennoch ist es für das spätere Dingfestmachen der Folgerungsbeziehung wichtig zu verstehen, welcher Art die Typ 1-Prozesse zu denken sind, die hier ablaufen. Es ist diese bestimmte Struktur der Prozesse, die sie als Prozesse der Intuition individuiert und somit eine Erkenntnisart etabliert, auf welche das Subjekt in Folge Bezug nehmen kann. 
Wie bereits plausibilisiert wurde (Kap. 3.4), lässt sich der Typ 1-Prozess, der von den mentalen Modellen zu Intuitionen ${ }_{\mathrm{NP}}$ führt, als Prozess des $A b$ lesens verstehen. Der Ausdruck >Ablesen< veranschaulicht hier, dass die Informationen bereits im mentalen Modell vorhanden sind, jedoch meist implizit, und dadurch ein weiterer kognitiver Vorgang nötig wird, um sie etwa für eine Problemlösung fruchtbar zu machen. Dabei ist der in diesem Zusammenhang metaphorische Charakter des Ausdrucks >Ablesen< zu betonen, denn die entsprechenden kognitiven Vorgänge können sich sowohl durch bewusstes, schrittweises Ableiten als auch durch unbewusste, schnelle Informationsverarbeitung ereignen. Wenn ich im Folgenden für beide Fälle davon spreche, dass ein Subjekt ein mentales Modell abliest, soll immer in Erinnerung behalten werden, dass es sich um einen Typ 1-Vorgang handeln kann, der keine bewusste Tätigkeit des Subjekts, keine Propositionalität und somit auch kein Ablesen im wörtlichen Sinn beinhaltet. Wer in Bezug auf Rechtfertigungsbeziehungen nichtbegrifflichen Gehalten, unbewussten Vorgängen und generell dem Typ 1-Denken skeptisch gegenüber steht, soll sich nicht an der Verwendung des Ausdrucks >Ablesen< stören, denn es sei zugegeben, dass der hier beschriebene Vorgang in der Regel keine Kontrolle des Subjekts beinhaltet und daher noch keine genuinen Gründe für daraus erwachsen können.

Die Struktur des >Ablesens soll dennoch genauer beschrieben werden, mit folgender Zielsetzung: Eingedenk der zuvor erwähnten Einschränkungen in Bezug auf die Gründe-Relation lassen sich zumindest folgerungsähnliche Verhältnisse bereits auf der Ebene des Typ 1-Ablesens von Intuitionen ${ }_{N P}$ aus mentalen Modellen feststellen. Im Prinzip ist alles, was dann noch fehlt, dass diese folgerungsähnlichen Verhältnisse auch unter die volle Kontrolle des Subjekts kommen, damit sie zu genuinen Folgerungsverhältnissen werden. Dies soll mit den weiteren Elementen des Modells ermöglicht werden. Sobald wir genuine Folgerungsverhältnisse haben, können wir von intuitiver Rechtfertigung im Vollsinn sprechen.

Zum besseren Verständnis der Metapher des Ablesens kann diese in Bezug auf zwei Beispiele veranschaulicht werden. Kehren wir zunächst zum Cartesischen »ich denke, ich existiere« zurück. Ich habe bereits dafür plädiert, dass die intuitive Überzeugung sich nicht in >ich denke< erschöpft, sondern sich auf das Folgen des >ich denke< aus >ich existiere $<$ bezieht. Dies ist das korrekte Verständnis in Bezug auf Descartes, da er in den Meditationen die Proposition >ich denke< bereits explizit nennt und es um das Intuitive im Schluss von >ich denke $<$ zu >ich existiere $<$ geht. Durchaus ist aber auch ein einfacherer Fall denkbar: Ein Subjekt hat die Überzeugung >ich existiere < und erlebt diese mit den phänomenalen Merkmalen der Unmittelbarkeit und Gewissheit; S ist 
also intuitiv überzeugt, dass sie existiert. Nun ist es - wie Descartes' auf der Metaebene des Philosophen anzusiedelnde Analyse plausibilisiert - denkbar, dass das Subjekt auf diese seine Existenz durch Typ 1-Denken, ausgehend vom Bewusstsein des eigenen Denkens, gekommen ist. Der Fall lässt sich plausibel so beschreiben: Das Subjekt hat ein mentales Modell seines eigenen Denkens. Dieses mentale Modell kann durch S' Typ 1-Denken jederzeit »abgelesen« werden, wobei eine dieser Ablesungen dem im mentalen Modell bestehenden Zusammenhang zur Existenz folgt: Wie ein mentales Modell eines Gebäudes implizites Wissen enthält, etwa hinsichtlich eines bestehenden Gebäudefundaments, enthält das mentale Modell >ich denke< implizites Wissen hinsichtlich der eigenen Existenz. Somit kann >ich existiere als begriffliche Transformation einer entsprechenden Intuition ${ }_{\mathrm{NP}}$ bezeichnet werden, die aus dem mentalen Modell des eigenen Denkens durch Typ 1-Denken abgelesen wurde.

Auch in Beispielen, die typischerweise in der psychologisch-kognitionswissenschaftlichen Intuitionsforschung diskutiert werden, lässt sich erkennen, wie Subjekte mittels Ablesen der mentalen Modelle auf Intuitionen kommen. Unser Beispiel der Feuerwehrkommandantin ist ein charakteristischer Fall dafür: In einer einflussreich gewordenen Studie, die den Natural Decision Making-Ansatz begründete, haben Gary Klein und Mitarbeitende die intuitiven Entscheidungen von Feuerwehrleuten untersucht. Hierbei stellten sie fest, dass in der überwiegenden Anzahl der Fälle keine längeren Abwägungen getroffen und beispielsweise mehrere Handlungsoptionen miteinander verglichen wurden, sondern vielmehr die jeweilige Situation als typisch für einen bestimmten Situations-Prototyp eingeordnet wurde, worauf ein Handlungsverlauf gewählt wurde, der diesem Prototyp entspricht. ${ }^{249}$ Dieser Prototyp entspricht einem mentalen Modell, welches Experten durch ihre Erfahrung entwickelt haben und mit dem sie die neuen Erfahrungen jeweils abgleichen. Wie das mentale Modell eines Descartes-ähnlichen Subjekts von seinem denkenden Ich auch seine Existenz beinhaltete, beinhaltet Claires mentales Modell für ein bestimmtes Ensemble von Bedingungen die Repräsentation des baldigen Einstürzens des Gebäudes. Wiederum ist entscheidend, dass diese Informationen nicht in propositionaler Form konstituiert sind, sondern in einem ikonischen mentalen Modell als strukturierte nichtbegriffliche Erfahrungsgehalte vorliegen. Im Gefahrenfall kommt es zu einer schnellen, via Typ 1-Prozesse verlaufenden Prüfung, inwiefern die aktuelle Situation diesem Einsturzgefahr-Modell entspricht. Daraus resultiert die Intuition ${ }_{N P}$, die eine Neigung zur Überzeugung konstituiert, dass das Gebäude bald einstürzen wird.

249 Vgl. Klein/Calderwood/Clinton-Cirocco (2010). 
In Claires Fall wird aufgrund dieser Neigung tatsächlich eine entsprechende intuitive Überzeugung gebildet. 250

Zusammenfassend wird ersichtlich, dass das Typ 1-Denken durch Ablesen mentaler Modelle auf Intuitionen ${ }_{\mathrm{NP}}$ kommen kann. Die Intuitionen $\mathrm{NP}_{\mathrm{N}}$ sind durch ihren Bezug zu mentalen Modellen und den Prozess des Ablesens zentraler Bestandteil der intuitiven Rechtfertigung. Was uns aber noch fehlt, sind die Elemente, die in der intuitiven Rechtfertigung einen Grund aufdecken können, der ein genuiner Grund für das Subjekt ist. Diese werden mit dem dritten und insbesondere dem vierten Element des Modells intuitiver Rechtfertigung gegeben.

(3) Der Gehalt der intuitiven Überzeugung ist identisch mit dem Gehalt der Intuition $_{\mathrm{NP}}$.

Dieses Element des Modells stützt sich auf den Zusammenhang zwischen Intuitionen $_{\mathrm{NP}}$ und intuitiven Überzeugungen (siehe oben, Kap. 3.2). Wie gezeigt wurde, konstituieren Intuitionen ${ }_{N P}$ Neigungen zu Überzeugungen. Das heißt, wenn ein Prozess der Überzeugungsbildung in Gang gesetzt wird und keine Anfechtungsgründe bestehen, wird das Subjekt die der Intuition ${ }_{\mathrm{NP}}$ entsprechende Überzeugung bilden. Was heißt hier >entsprechend<? Ich schlage vor, dies ganz einfach als Übereinstimmung der Gehalte zu verstehen, wobei der Gehalt der Intuition ${ }_{\mathrm{NP}}$ für das Subjekt zunächst nichtbegrifflich und erst der Gehalt seiner entsprechenden Überzeugung begrifflich vorliegt.

Am Beispiel des Spielerfehlschlusses sei dieser Vorschlag veranschaulicht: In einem mentalen Modell des Subjekts ist die Information, die den Gehalt der Spielerfehlschluss-Intuition konstituiert, bereits enthalten. Diese Information wird durch Typ 1-Denken aus dem mentalen Modell abgelesen, wenn Anlass dazu besteht (so etwa, wenn das Subjekt an einem Würfelspiel teilnimmt). Das Ergebnis dieser Ablesung ist in unserem Verständnis die Intuition ${ }_{\mathrm{NP}}$. Es ist nun möglich, aber nicht notwendig, dass das Subjekt sich seiner Intuition bewusstwird und eine entsprechende intuitive Überzeugung bildet, etwa der Form:

250 Für sehr schnelle Entscheidungen ist Kleins Modell - auch unter dem Titel Recognition Primed Decision Making bekannt - in jüngerer Zeit in die Kritik geraten. So schlagen Baber, Chen und Howes für diese Fälle als bessere Alternative ein Modell vor, das den Fokus weg von deklarativem und hin zu prozeduralem Wissen verschiebt. In den entsprechenden Situationen, in denen schnelle Entscheidungen getroffen werden, sind Experten dieser Alternative zufolge auf bestimmte Wahrnehmungsreize primed, wodurch sie diese herausfiltern und darauf in einer trainierten Weise reagieren können (vgl. Baber/ Chen/Howes 2015). Eine solche Alternative stellt für den hier dargestellten Ansatz des intuitiven Schließens kein Problem dar, da in einem solchen Fall wahrscheinlich nicht mehr sinnvoll gesagt werden kann, dass das Subjekt eine intuitive Überzeugung hat; geschweige denn, dass es eine Intuition als Grund nimmt. Vielmehr gleicht der Fall in der entsprechenden Hinsicht Reiz-Reaktions-Kopplungen. 
»Wenn etwas hier oft vorgekommen ist, wird es beim nächsten Mal weniger wahrscheinlich vorkommen « (’hier bezieht sich auf die aktuelle Situation, also auf das Würfelspiel). Das Subjekt verfügt in diesem Fall über Begriffe, welche den Gehalt der Intuition erfassen und wendet diese Begriffe auch an.

Das dritte Element des Modells intuitiver Rechtfertigung postuliert, dass in jedem Fall, in dem eine intuitive Überzeugung vorliegt, auch eine entsprechende nichtpropositionale Intuition vorliegt, deren Gehalt identisch mit dem Gehalt der fraglichen Überzeugung ist. Der wohl entscheidende Grund für eine solche Annahme liegt in einem Konjunktivismus, der uns erlaubt, dieselben fundierenden Typ 1-Denkprozesse in einem Subjekt zu vermuten, welches eine intuitive Überzeugung bezüglich des Spielerfehlschlusses hat, wie in einem Subjekt, welches einfach aufgrund einer Spielerfehlschluss-Intuition handelt, ohne die entsprechende Überzeugung zu haben. Zu vermuten ist, dass ein solcher Konjunktivismus auch in entsprechenden psychologischen Experimenten überprüft werden könnte und in Hirn-Scans wohl dieselben Prozesse festgestellt würden, wenn auch im Fall der Überzeugung gewisse andere Prozesse hinzukommen. Dies zu belegen wäre eine empirische Aufgabe, doch zumindest ist es sehr plausibel anzunehmen, dass wir diese Gemeinsamkeit der Typ 1-Prozesse feststellen würden. Da die Typ 1-Prozesse als Ablesungen aus mentalen Modellen bestimmt wurden, die zu Intuitionen $\mathrm{NP}_{\mathrm{N}}$ führen, ist die naheliegendste Konsequenz, dass der Gehalt dieser Intuitionen $_{N P}$ identisch mit dem Gehalt einer intuitiven Überzeugung ist, zu der das Subjekt durch die Intuition $_{\mathrm{NP}}$ geneigt ist.

(4) S ordnet durch mentale Demonstration die Überzeugung als intuitive Überzeugung ein.

Wir erleben im Fall einer intuitiven Überzeugung das Vorhandensein einer Überzeugung, die wir nicht mit einer deduktiven Begründung herleiten können und die zugleich von Unmittelbarkeit und Gewissheit begleitet ist. Diese Erlebnisqualität ist ein entscheidendes Element, um überhaupt von einer intuitiven Überzeugung sprechen zu können. Hat ein Subjekt eine Überzeugung völlig ohne dieses Erleben, entspräche dies aus der Subjekt-Perspektive einer auf rätselhafte Weise zustande gekommenen Eingebung. Diese könnte zwar zuverlässig sein, ein Subjekt wäre aber irrational, wenn es dieser Eingebung einfach folgen würde, insbesondere dann, wenn es über gegenläufige Evidenz verfügt. ${ }^{251}$ Es kann nichts anderes als die Erlebnisqualität sein, die dem Subjekt anzeigt, dass es sich nicht etwa um ein bloßes Raten oder eine andere irrationale Überzeugung handelt, denn durch nichts anderes unterscheidet sich die

251 BonJours Beispiel des Hellsehers Norman (Kap. 4.3) kann als solcher Fall eingeordnet werden. 
Überzeugung aus der Perspektive des Subjekts von solchem. Somit lässt sich davon ausgehen, dass es die erwähnte Erlebnisqualität der intuitiven Überzeugung, also deren phänomenalen Eigenschaften der Unmittelbarkeit und Gewissheit sind, die das Subjekt diese Überzeugung als intuitive Überzeugung einordnen lässt.

Im Anschluss an diesen Befund ist nun zu klären, wie diese Einordnung der Überzeugung vor sich geht. Zur Beantwortung dieser Frage wird das Konzept der mentalen Demonstration dienen. Im Zusammenspiel mit den anderen Elementen des Modells führt die mentale Demonstration dazu, dass ein Subjekt über genuine Gründe für intuitive Überzeugungen verfügt. Dies soll im Folgenden ausgeführt werden.

Das Subjekt ordnet die aktuelle intuitive Überzeugung aufgrund deren phänomenalen Eigenschaften als Vorkommnis der Intuition ein. An das vierte Element des Models ist also ein Wissen des Subjekts in Bezug darauf geknüft, was Intuition ist. Hierbei ist es wichtig zu präzisieren, was dieses Wissen beinhaltet und v.a. nicht beinhaltet. Keineswegs soll postuliert werden, dass das Subjekt das Wort >Intuition< kennt oder hier in Anschlag bringt. Auch ist keinerlei Wissen über die kognitiven Prozesse, die der Intuition zugrunde liegen (also etwa über das hier beschriebene Typ 1-Denken, das Intuitionen aus mentalen Modellen abliest) verlangt. Vielmehr geht es um ein rudimentäres Wissen über die Art des mentalen Zustands: Das Subjekt muss diesen Zustand wiedererkennen. Es muss feststellen können, dass es diesen Zustand schon öfter erlebt hat, um dessen Gehalt entsprechend einordnen zu können. Dies ist entscheidend dafür, dass in Zusammenhang mit dem Zustand Gründe in Anschlag gebracht werden können. Dass diese Einordnung aufgrund der phänomenalen Eigenschaften des Zustands möglich ist, wurde bereits aufgezeigt (siehe Kap. 3.1).

Um die These zu stützen, dass die Einordnung des mentalen Zustands seitens des Subjekts notwendig für eine Begründungsrelation ist, lässt sich eine Analogie zur visuellen Wahrnehmung herstellen: Wenn ich etwas sehe, kann der Gehalt meiner visuellen Wahrnehmung nur dann begründende Kraft haben, wenn die Repräsentation des Gehaltes von einem rudimentären Bewusstsein über den Zustand des Sehens begleitet ist. Ich muss wissen, was es heißt zu sehen - etwa durch vorgängige Erfahrungen -, damit ich um die begründende Kraft einer derartigen Erfahrung weiß. Um diese begründende Kraft wiederum muss ich wissen, wenn der Grund ein Grund für mich sein soll. Ich habe gelernt, dass diese Art der Bezugnahme auf die Welt erfolgsversprechend ist und ich Überzeugungen aufgrund der Wahrnehmung bilden darf. Dasselbe gilt für die Intuition. Ich kann keine intuitiven Gründe haben, wenn ich keinerlei Bezug zur Intuition als Erkenntnisart habe, d.h. diese Erkenntnisart nicht 
kenne. Intuitionen ${ }_{\mathrm{NP}}$ können dann zwar wirken, indem sie z.B. direkt in Handlungen umgesetzt werden, sie können aber keine Gründe für das Subjekt sein, wie dies für die hier untersuchte epistemische Rechtfertigungsbeziehung zwischen Intuitionen $_{\mathrm{NP}}$ und intuitiven Überzeugungen erforderlich ist.

Für die Frage der Rechtfertigung zentral ist, dass Urteile, die sich auf Bekanntschaft beziehen, stark gerechtfertigt (wenn auch nicht infallibel) sind. ${ }^{252}$ Der Grund dafür ist, dass ich die phänomenalen Eigenschaften aufgrund der Bekanntschaft auf eine einzigartig direkte Weise erfassen kann. So ist im Fall der aktuell auftretenden Intuition mein Urteil, dass ich jetzt etwas auf unmittelbare und Gewissheit vermittelnde Weise erlebe, kaum bezweifelbar. Mit anderen Worten: Man kann sich kaum darüber täuschen, eine Intuition zu haben. Diesem Befund muss aber sogleich präzisierend hinzugefügt werden: Unsicherheiten über die korrekte Anwendung des Begriffs >Intuition< (oder gar gänzliche Unkenntnis dieses Begriffs) sind möglich. Doch wenn ein Subjekt aufgrund der Bekanntschaft mit genau den richtigen phänomenalen Eigenschaften einen mentalen Zustand individuiert, dann kann es sich in dieser Individuierung kaum irren. Auf welche Weise wir mit den phänomenalen Eigenschaften eines mentalen Zustands wie der Intuition bekannt sein können, lässt sich nun genauer bestimmen. Außerdem kann gezeigt werden, mit welchen weiteren Schritten diese Bekanntschaft dazu führt, dass das Subjekt eine aktuell erlebte Intuition auch als solche einordnen kann. Diese Einordnung ist wichtig, denn durch sie steht etwas zur Verfügung, was das Subjekt als Grund einspannen kann.

Mentale Demonstration ist der entscheidende Vorgang, der die Brücke von der Bekanntschaft mit den phänomenalen Eigenschaften der intuitiven Überzeugung zu einer Verfügbarkeit der Intuition als Grund schlägt. Frank Hofmann bestimmt die mentale Demonstration in Bezug auf introspektive Erkenntnis wie folgt:

By mental demonstration we are able to bring the property contents of our experiences into the realm of (conceptual) thinking in such a way as to make possible the application of ordinary conceptual classificatory abilities. [...] Suppose, for example, that Fred is looking at a red expanse and is having an experience as of something red. He experiences a property, a particular shade of red (call it $>$ red $37<$ ). By mental demonstration he can then refer in his mind to this property - the property he is experiencing - as >this (or as >this property $<$ ). By applying a demonstrative concept $>$ this $<$ he can start thinking about the property that he is experiencing. 253

252 Vgl. Gertler (2011, S. 12).

253 Hofmann (2019, S. 1310). 
In Einklang mit dem bisher Postulierten können wir davon ausgehen, dass mentale Demonstration auch in Bezug auf die phänomenalen Eigenschaften intuitiver Erkenntnis möglich ist. Faktisch nahezu untrennbar, aber doch analytisch von der mentalen Demonstration zu trennen ist der nächste Schritt, in welchem das Subjekt seine begrifflichen Fähigkeiten in Bezug auf den durch mentale Demonstration festgehaltenen phänomenalen Aspekt in Anschlag bringt.

Beide Schritte zusammenfassend lässt sich der Vorgang in Bezug auf intuitive Erkenntnis etwa wie folgt paraphrasieren: »Ich erlebe diese Art eines mentalen Zustands«, wobei >diese Zustands verweist, also auf Unmittelbarkeit und Gewissheit). Ebenfalls bereits durch die Paraphrase angesprochen wird der dritte Aspekt, der mit mentaler Demonstration und begrifflicher Klassifikation einhergeht: Das Einfließen von Hintergrundwissen, welches sich durch die Einführung weiterer Begriffe zeigt. ${ }^{254} \mathrm{Im}$ angesprochenen Fall verfügt das Subjekt beispielsweise über den Ausdruck >mentaler Zustand ( (sie ist wahrscheinlich eine Philosophin). Ein anderes Subjekt könnte den erlebten mentalen Zustand auch bloß als >Gedanke< oder 〉Gefühl bezeichnen. In beiden Fällen können die Subjekte mit ihrem jeweiligen Ausdruck aber auf denselben mentalen Zustand, also die Intuition referieren. Wenn die Intuition als Grund dienen soll, muss eine Einordnung des Erlebten als Intuition möglich sein, mit welchem Ausdruck auch immer sie benannt wird.

Mittels mentaler Demonstration gelingt es also einem Subjekt, seine Intuition als solche zu verstehen. Die Grundidee dieses Vorschlags ist, dass sich all die begründende Kraft der Intuition, die maßgeblich durch Vorgänge auf der nichtpropositionalen Ebene des Typ 1-Denkens konstituiert wird, auch durch das Subjekt als solche begründende Kraft verstehen lässt und dadurch unter seine Kontrolle geraten kann, was eine Begründung im Vollsinn ermöglicht. Dabei ist keine Einsicht in die Vorgänge des Typ 1-Denkens notwendig. Die mentale Demonstration, verbunden mit den weiteren ausgeführten Elementen, sorgt dafür, dass das Subjekt genau das herausgreifen kann, was eine Intuition ausmacht. Dies ist für den internalistischen Aspekt der Begründung entscheidend.

Wir standen vor dem Problem, inwiefern Intuitionen, die sich maßgeblich durch Typ-1-Denken konstituieren, Gründe für intuitive Überzeugungen liefern können, die echte Gründe für Subjekte sind. Um dieses Problem zu lösen, wurde das Modell intuitiver Rechtfertigung vorgeschlagen, welches vier Elemente enthält, die im Zusammenspiel die internalistische Forderung

254 Vgl. Hofmann (2019, S. 1306). 
an Gründe zu erfüllen vermögen. Abschließend soll verdeutlicht werden, wie dieses Zusammenspiel aussieht. Hierzu ist es sinnvoll, auf die Relation der Bekanntschaft zu fokussieren. Bekanntschaft kommt im Modell intuitiver Rechtfertigung zweimal ins Spiel: Erstens erkennt das Subjekt, wie soeben dargelegt, mittels phänomenaler Merkmale die intuitive Überzeugung als intuitiv (4). Dies schlägt die erste Brücke von den Typ 1-Denkvorgängen, die quasi im Untergrund wirken, zur Oberfläche, auf der das Subjekt steht. Zweitens ist das Subjekt via mentale Modelle mit dem Sachverhalt bekannt, welcher den Gehalt der Intuition erfüllt (1). Dies schlägt die zweite Brücke von den Typ 1Denkvorgängen zur Oberfläche des Subjekts. Die zweite Brücke ergänzt die erste in notwendiger Weise, weil dadurch auch dasjenige, worauf sich die Intuition richtet, also der Gehalt der Intuition, dem Subjekt verfügbar wird. Somit ist das Subjekt sowohl mit der Art seines mentalen Zustands als auch mit dem dadurch Erkannten bekannt. Beides ist ihm verfügbar und konstituiert gemeinsam den Grund des Subjektes für seine intuitive Überzeugung. Das Modell intuitiver Rechtfertigung kann somit zeigen, wie Intuitionen $n_{\mathrm{NP}}$ Gründe für Subjekte sein können. Da weiter eine vollständige Theorie intuitiver Rechtfertigung auch den externalistischen Aspekt, etwa die Beurteilung der Zuverlässigkeit des Typ 1-Denkens eines Subjekts, beinhaltet, kann diese vollständige Theorie die Möglichkeit intuitiver Rechtfertigung aufweisen.

\subsubsection{Ein Rückfall in den Mythos des Gegebenen?}

Die Ausführungen des Modells intuitiver Rechtfertigung sollen mit zwei Erwiderungen auf mögliche Einwände abgeschlossen werden. Hier geht es zuerst um einen drohenden Rückfall in den »Mythos des Gegebenen «. Im folgenden Unterkapitel wird das Problem der angeblichen inferentiellen Isolation nichtbegrifflicher Gehalte besprochen.

Wie bereits erwähnt wurde, ist ein Ansatz, der Gründe zu einem maßgeblichen Teil auf der nichtpropositionalen Ebene verorten will, dem Einwand ausgesetzt, dass es sich hier um keine genuinen Gründe für ein Subjekt handeln kann. Dies wird damit begründet, dass der Ansatz auf ein außerhalb des Bereiches der Begrifflichkeit liegendes Gegebenes rekurriert, Gründe aber nur im Bereich der Begrifflichkeit genuine Gründe für ein Subjekt sein können. Dies war McDowells Kritik an der nonkonzeptualistischen Position Evans' im Bereich der Philosophie der Wahrnehmung. Ist das vorgestellte Modell intuitiver Rechtfertigung gegen diese Kritik immun oder lässt sich gegen dieses Modell der Vorwurf vorbringen, es handle sich letztlich um eine weitere Theorie des Gegebenen?

Eine Antwort auf diese Frage muss zwiespältig ausfallen: Gehen wir die konzeptualistische Kritik an Theorien des Gegebenen einerseits und die 
Ausführungen zur intuitiven Rechtfertigung andererseits nochmals durch, so lässt sich eine wohl nicht überwindbare Divergenz im Verständnis von >Grund < feststellen. So soll den konzeptualistischen Kritikern gegenüber zugegeben werden, dass in einem ambitionierten Sinn von >Grund $<$ das hier vorgebrachte Modell keine genuinen Gründe zu liefern vermag. Es überrascht daher nicht, wenn sie das Modell weiterhin als Theorie des Gegebenen bezeichnen. Zugleich kann aber aufgrund der Ausführungen zur intuitiven Rechtfertigung mit Fug und Recht ein entspannteres Verständnis von >Grund etabliert werden, welches der internalistischen Gründe-Erfordernis Genüge tut.

Die Unterscheidung der beiden Verständnisse von >Grund< knüpft an der Unterscheidung zwischen Verfügbarkeit und Zugänglichkeit an (siehe Kap. 4.3). Aufschlussreich ist die Feststellung, inwiefern diese Unterscheidung mit dem jeweils vorherrschenden philosophischen bzw. psychologischkognitionswissenschaftlichen Verständnis der Gründe-Relation übereinstimmt. So lässt sich feststellen, dass Psychologen keine Schwierigkeiten damit haben, Gründe-Relationen auf der Ebene subpersonaler Prozesse dingfest zu machen, wie die entsprechenden Fachtermini belegen (intutive reasoning, system 1-reasoning, unconscious reasoning, usw.). Philosophinnen hingegen sträuben sich gegen ein solches Gründe-Verständnis, da es Kerngehalte klassischer Begründungslehren verletzt und dadurch das Verständnis von >Grund so sehr verwässert, dass der Terminus inhaltsleer zu werden droht. Dazu Paul Boghossian:

Suppose some reasoning was such that, having rehearsed the premises, some conclusion simply came to you (accompanied perhaps by the feeling that it is >right<), but not accompanied by any awareness of the process leading up to it. Full rationality would require that one ask oneself whether to endorse the conclusion that has simply come to you in this way. And this in turn would require that you lay bare the reasoning process by which the premises are supposed to have led to the conclusion. In other words, full rationality requires that a selfaware process of reasoning, one with no blind spots in it, vet the deliverances of a System 1 process and rule on their correctness. ${ }^{255}$

Die philosophische Motivation hinter diesen Anforderungen wird von Boghossian ebenfalls klar herausgearbeitet. Er bezeichnet sie als taking condition:

Inferring necessarily involves the thinker taking his premises to support his conclusion and drawing his conclusion because of that fact. The intuition behind the Taking Condition is that no causal process counts as inference, unless it

255 Boghossian (2014, S. 16). 
consists in an attempt to arrive at a belief by figuring out what, in some suitably broad sense, is supported by other things one believes. In the relevant sense, reasoning is something we do, not just something that happens to us. And it is something we do, not just something that is done by sub-personal bits of us. ${ }^{256}$

Boghossians Ausführungen bringen die klassische philosophische Haltung bezüglich der Begründungsrelation auf den Punkt. Dies ermöglicht es, auch die Kritik an dieser Haltung zielgerichtet vorzubringen. In den oben zitierten Passagen scheint ein gewisser Übergang stillschweigend vorgenommen $\mathrm{zu}$ werden. Machen wir diesen explizit, gelangen wir zu der beabsichtigten Unterscheidung der zwischen den beiden Gründe-Verständnissen.

Bei dem problematischen Übergang handelt es sich um denjenigen in Bezug auf die Ausdrücke inferring und reasoning. Ich gehe davon aus, dass sich diese Begriffe mit >schließen ` (inferring) bzw. >begründen` (reasoning) sinnvoll übersetzen lassen. Für >schließen < besteht in der philosophischen und psychologischen Literatur weitgehend Einigkeit, dieses im Bereich des expliziten Umganges eines Subjekts mit Propositionen zu verorten. ${ }^{257}$ Demnach sind Ausdrücke wie >intuitives Schließen< missverständlich oder gar widersinnig, weshalb sie hier vermieden werden. Für >begründen ২ hingegen gehen das philosophische und das psychologische Verständnis auseinander. Während Philosophinnen dazu tendieren, Begründungsverhältnisse vollständig im Bereich des Schließens, also auf der propositionalen Ebene zu verorten, verwenden Psychologen den Begründungsbegriff (reasoning) auch in Bezug auf die subpersonale Ebene. ${ }^{258}$

256 Boghossian (2014, S. 5).

257 Siehe die Einträge zu inference in den oxford dictionnaries für Philosophie und Psychologie im Vergleich:

Philosophie: »The process of moving from (possibly provisional) acceptance of some propositions, to acceptance of others« (Blackburn 2016).

Psychologie: »Reasoning from premises to conclusions; or a conclusion arrived at by this process. (Colman 2015).

$25^{8}$ Siehe dazu« die Einträge zu reasoning in den oxford dictionnaries:

Philosophie: »Any process of drawing a conclusion from a set of premises may be called a process of reasoning « (Blackburn 2016).

Psychologie: »Cognitive processing directed at finding solutions to problems by applying formal rules of logic or some other rational procedure« (Colman 2015).

Dass Psychologinnen und Psychologen solche rationalen Verfahren auch auf der subpersonalen Ebene verorten, zeigen bereits die Titel der Werke wie How we reason (Johnson-Laird 2006) oder Kahnemans unterdessen weit verbreitete Unterscheidung zwischen einer schnellen (intuitive) und einer langsamen (reflektive) Art des Denkens (vgl. Kahneman 2012). 
Als Philosoph geht Boghossian im obigen Zitat ohne weitere Erklärung direkt von inference zu reasoning über. Was ihn dazu zu berechtigen scheint, ist die taking condition. Für das Schließen ist diese unkontrovers, wie wir nach dem obigen Vergleich einräumen können. Doch gilt sie auch für die Gründe-Relation? Aufgrund der bisherigen Ausführungen in dieser Arbeit muss die Antwort darauf lauten:Ja und nein. Entsprechend des Unterschiedes zwischen >Gründe haben und >Gründe geben gelten zwei Arten von taking. Mein Zugriff auf den Grund muss explizit und propositional verfasst sein, wenn ich diesen vollständig artikulieren muss, etwa in einer Diskussion, in der ich meine Position argumentativ verteidige. Der Grund wird damit für mich und für andere vollständig zugänglich und nachprüfbar, ganz im Sinne der full rationality im ersten Zitat Boghossians. Dies ist der strenge Sinn von taking. Um einen Grund zu haben, der auch aus internalistischer Perspektive genuin, da mein Grund ist, reicht hingegen die Verfügbarkeit des Grundes aus. In dieser Hinsicht ist die lateinisch-englische Begrifflichkeit (reasoning) hilfreicher, da sie die Verbindung zur Rationalität aufrechterhält. Wir müssen uns letztlich fragen, ob es rational ist, dass ein Subjekt eine bestimmte intuitive Überzeugung fasst. Die Ausführungen zum Modell der intuitiven Rechtfertigung haben hinreichend gezeigt, dass diese Rationalität der intuitiven Überzeugung auch im internalistischen Sinn gegeben sein kann, da die Verfügbarkeit des Grundes für das Subjekt (maßgeblich durch Bekanntschaft) gewährleistet ist, auch wenn dieser Grund auf der Ebene des Typ 1-Denkens konstituiert wird.

Mit diesen Erkenntnissen komme ich auf den Vorwurf zurück, es handle sich auch bei dem Modell der intuitiven Rechtfertigung um eine Theorie des Gegebenen, die somit keine genuine Gründe-Relation etablieren könne. Die Antwort auf diesen Vorwurf muss aufgrund der obigen Ausführungen zwiespältig ausfallen. Gehen wir von einem traditionellen Gründe-Verständnis aus, welches Gründe nur im Bereich des Schließens zulässt, würde jeder Versuch, nichtpropositionale Elemente in eine Rechtfertigungstheorie einbauen zu wollen, in die Falle des Gegebenen tappen. Lassen wir aber ein offeneres Gründe-Verständnis im Sinn des reasoning zu, kann die Kritik zurückgewiesen werden. Um einen Grund für meine intuitive Überzeugung zu haben, muss dieser Grund nicht propositional verfasst sein. Die Bekanntschaft ist dem begrifflichen Zugang auf Propositionen hier ebenbürtig, wenn die Bezüge zwischen Bekanntschaft und Rechtfertigung sorgfältig ausgearbeitet werden. Diesorgfältige Ausarbeitung ist insofern wichtig, als derungenügend reflektierte Verweis auf Bekanntschaft allein noch keine Gründe-Relation (auch nicht im offeneren Sinn) etablieren kann. Ungenügend wäre es etwa, sich mit dem Element der Bekanntschaft mit dem Sachverhalt zufriedenzugeben. Dies wäre 
tatsächlich eine klassische und daher problematische Variante einer Theorie des Gegebenen, die den Grund für die intuitive Überzeugung in der unmittelbaren Erfahrung der Tatsache sucht, die durch diese Überzeugung ausgedrückt wird. ${ }^{259}$ Das Modell intuitiver Rechtfertigung hingegen enthält die Bekanntschaftsrelation nicht in dieser kruden Weise, sondern fügt Elemente hinzu, welche in Kombination eine Zurückweisung dieses Modells als Theorie des Gegebenen erschweren. Die Zurückweisung bleibt aber möglich, wenn man sich auf das klassische, also inferentielle Verständnis von Begründung berufen möchte. Keine auch noch so sorgfältige Ausarbeitung nichtpropositionaler Gründe-Relationen kann dies verhindern.

\subsubsection{Zum Problem der inferentiellen Isolation}

Kernanliegen der bisherigen Ausführungen war aufzuzeigen, inwiefern Intuitionen $_{\mathrm{NP}}$, eingebettet in ein Modell intuitiver Rechtfertigung, zu einem maßgeblichen Teil intuitive Überzeugungen begründen können. Mit der Annahme der internalistischen Forderung wurde eine erste Adäquatheitsbedingung für Rechtfertigung ausgedrückt:

(AV): Gründe müssen für das Subjekt verfügbar sein.

In der Ausarbeitung des Modells intuitiver Rechtfertigung wurde (AV) im Wesentlichen durch den mehrfachen Einfluss der Bekanntschaftsrelation Rechnung getragen.

Nun ist ein entscheidender Einwand gegen das Vorgebrachte zu prüfen: Gemäß verbreiteter Auffassung sind nichtbegriffliche Gehalte inferentiell isoliert, d.h., sie können nicht zu anderen Gehalten in eine rechtfertigende Relation treten. ${ }^{260} \mathrm{Ob}$ eine solche Isolation auch dann gilt, wenn die Rechtfertigungen über ein Modell wie das vorgestellte vermittelt wird, ist nicht zum Vornherein klar. Würden wir die These der inferentiellen Isolation nichtbegrifflicher Gehalte im starken Sinn interpretieren, also so, dass auch unter Zuhilfenahme solcher Vermittlungen die Rechtfertigung nicht von nichtbegrifflichen Gehalten ausgehen kann, stünde sie in offensichtlichem Widerspruch zu den bis-

259 Vgl. Schildknecht (2002, S. 164).

26o Vgl. Schildknecht (2002, S. 184) - Zu beachten ist hier das Verständnis von >inferentiell<, das nicht den Ausführungen im vorigen Kapitel entspricht. Wenn wir inference mit >schließen< übersetzen, sind wir bei der strengen, also auf Propositionalität festgelegten Version von Begründung. Die Möglichkeit, dass nichtbegriffliche Gehalte in inferentiellen Relationen stehen, wäre damit bereits a priori ausgeschlossen und die Diskussion über die inferentielle Isolation würde überflüssig. Das Problem geht auf die verbreitete Unschärfe in der Verwendung von inference und reasoning zurück. Ich werde der Einfachheit halber bei dem Ausdruck sinferentiell< bleiben, meine damit aber die allgemeine Gründe-Relation, also die Beziehung zwischen Gehalten im Sinne des reasoning. 
herigen Ausführungen. Im Folgenden soll daher, quasi sicherheitshalber, eine solche starke These inferentieller Isolation nichtbegrifflicher Gehalte zurückgewiesen werden. Dazu ist in einem ersten Schritt das mit dieser These verbundene Verständnis von Begründung herauszuarbeiten. Genauer: Welche Bedingung muss ein Gehalt - neben (AV) - erfüllen, um den Status eines Grundes zu erhalten? Anschließend kann gezeigt werden, dass Intuitionen ${ }_{\mathrm{NP}}$ als nichtbegriffliche Gehalte diese weitere Adäquatheitsbedingung durchaus erfüllen können (wenn nicht direkt, so doch als Bestandteile eines Modells intuitiver Rechtfertigung), womit der Einwand der inferentiellen Isolation nichtbegrifflicher Gehalte zurückgewiesen werden kann.

Die klassische Begründung für die inferentielle Isolation nichtbegrifflicher Gehalte läuft via negativa: So wird zunächst dafür argumentiert, dass sich begriffliche Gehalte wesentlich dadurch auszeichnen, dass sie in inferentiellen Relationen zu anderen Gehalten stehen. Dies wird zur These verstärkt, wonach diese inferentielle Einbettung geradezu das Markenzeichen begrifflicher Gehalte ist, wie etwa bei Tim Crane nachzulesen ist:

The notion of possessing a concept is then naturally explained as follows. To possess a concept is to be in intentional states whose inferential relations are an appropriate function of their contents. The elements in a thinker's network of intentional states are essentially inferentially related to one another. Concepts are the constituents required to explain these inferential relations. So a thinker could not be in the relevant intentional states unless they contain concepts. ${ }^{261}$

Die Begründung für diese Auffassung von inferentieller Relation als Markenzeichen für Begrifflichkeit ist nachvollziehbar: Erstens wird festgehalten, dass Begrifflichkeit via Begriffsbesitz zu definieren ist. ${ }^{262}$ Weiter wird argumentiert, dass nur dann von Begriffsbesitz gesprochen werden kann, wenn jemand verschiedene inferentiell aufeinander bezogene Zustände (z.B. Überzeugungen) mit diesem Begriff als Konstituenten haben kann. So verfügt ein Subjekt nur dann über den Begriff $>$ Käse $<$, wenn es ein Netz von inferentiell aufeinander bezogenen Überzeugungen in Bezug auf Käse hat (z.B. >Käse ist aus Milch hergestellt $<$, daraus folgt: $>$ Käse ist ein Lebensmittel $<) .{ }^{263}$ Der nächste Schritt, wonach nichtbegriffliche Gehalte diese inferentielle Einbettung nicht

261 Crane (1992, S. 147f.).

262 Für die Argumentation dafür siehe Crane 1992; sie läuft auf folgende Definition von begrifflichem Gehalt hinaus:

A state with conceptual content - e.g. a belief - is one such that the subject of that state has to possess the concepts that canonically characterise its content in order to be in that state.

(Crane 1992, S. 143)

263 Vgl. Crane (1992, S. 145). 
aufweisen, ist naheliegend, folgt aber nicht direkt aus den obigen Überlegungen. Hier fehlt noch die Argumentation, warum Begriffsbesitz nicht nur hinreichend, sondern auch notwendig ist um inferentielle Relationen zwischen Gehalten herzustellen. Diese Lücke lässt sich mit folgender Überlegung Gottlob Freges schließen, die einen Ursprung für die bisher erläuterten Überlegungen zur Deckungsgleichheit von Inferentialität und Begrifflichkeit darstellt:

Die Aufgabe unserer Volkssprachen ist wesentlich erfüllt, wenn die mit einander verkehrenden Menschen mit demselben Satze denselben Gedanken verbinden, oder doch annähernd denselben. Anders liegt die Sache, wenn Schlüsse gezogen werden sollen; denn dabei ist wesentlich, dass in zwei Sätzen derselbe Ausdruck vorkomme, und dass dieser in beiden genau dieselbe Bedeutung habe. Er muss also für sich eine Bedeutung haben, die unabhängig ist von den andern Theilen des Satzes. ${ }^{264}$

Frege zufolge ist Begrifflichkeit notwendige Bedingung für Inferentialität. Seine Forderung, wonach sich semantisch fixierte Konstituenten in zwei mentalen Zuständen wiederholen müssen, damit Folgerungen möglich sind, ist nur so zu verstehen, dass beide mentale Zustände denselben Begriff enthalten müssen. ${ }^{265}$ Diese Überlegungen führt Christiane Schildknecht weiter zu einer Definition für begrifflichen Gehalt:

(CC): An intentional state $S$ has a conceptual content iff a subject $X$ 's being in $S$ entails that $S$ has inferentially relevant constituents. ${ }^{266}$

(CC) impliziert, dass Zustände mit nichtbegrifflichen Gehalten nicht in inferentiellem Bezug zueinander stehen. Eine solche Auffassung von begrifflichem und nichtbegrifflichem Gehalt wird von nonkonzeptualistischer Seite etwa durch Raftopoulos und Müller unterstützt, die den nichtbegrifflichen Gehalt als kognitiv undurchdringbar (cognitively impenetrable) und durch bottom up-Wahrnehmungsprozesse zustande kommend charakterisieren. ${ }^{267}$

Die Fregesche Auffassung, wonach die Einbettung in ein Begriffsnetz (und somit Begrifflichkeit) notwendig dafür ist, dass ein Gehalt einen Grund darstellen kann, scheint auf eine Adäquatheitsbedingung der Wiederholung

264 Frege in einem Brief an Peano (29.9.1896), siehe Frege (1976, S. 183). Vgl. dazu Crane (1992, S. 146).

265 Vgl. Crane (1992, S. 147).

266 Schildknecht (2002, S. 184).

267 Vgl. Raftopoulos/Müller (2006, S. 210). 
zurückführbar zu sein, die für Inferentialität aufgestellt wird. Sie tritt zur bereits erfüllten Adäquatheitsbedingung der Verfügbarkeit (AV) hinzu:

(AW): In einer Schlussfolgerung müssen sich Elemente wiederholen.

Im Fall propositionaler Schlüsse ist die Richtigkeit von (AW) offensichtlich; kein gültiger Schluss kommt ohne die Wiederholung von Begriffen aus (z.B.: »Alle Menschen sind sterblich, Sokrates ist ein Mensch, Sokrates ist sterblich «). Hier werden die Begriffe >Mensch<, `Sokrates« und >sterblich was eine notwendige Bedingung dafür ist, dass zwischen den Sätzen Folgebeziehungen bestehen können. Doch ist (AW) nur mit der Begrifflichkeit der inferentiellen Komponenten zu haben? Dies folgt nicht zwingend. Die bei Frege ihren Ursprung nehmende und von nachfolgenden Positionen weitergeführte semantische Fixierung der sich wiederholenden Komponenten ist voreilig, denn sich wiederholende Komponenten mit fixiertem Gehalt im Sinne von (AW) sind auch im oben entworfenen Modell intuitiver Rechtfertigung vorhanden. Insofern ist zwar gemäß (AW) der Fregeschen Forderung der Wiederholung von Komponenten Rechnung zu tragen; dass diese Komponenten aber propositional verfasst sein müssen, ist ein konzeptualistische Vorannahme, nicht eine Folge aus (AW).

Inwiefern ist (AW) im hier vorgestellten Modell der intuitiven Rechtfertigung erfüllt? Hier ist der Bezug zu mentalen Modellen, deren Ikonizität eine Fixierung des Gehaltes ebenso zu garantieren vermag wie Begrifflichkeit, entscheidend. Dies lässt sich anhand der besprochenen Beispiele aufzeigen: Das ikonische mentale Modell der Feuerwehrkommandantin über die Brand-Gefahrensituation stellt die nichtbegrifflichen Elemente erstmals in inferentielle Bezüge zueinander: Wie in einem Gebäude Strukturen miteinander verbunden und dreidimensional angeordnet sind, repräsentieren im ikonischen Modell die Gehalte zeitlich, räumlich und kausal miteinander verbundene Elemente; beispielsweise die Windrichtung, Stärke des Feuers und Materialbeschaffenheit des Gebäudes. Diese mentale Repräsentation stellt Informationen zur Verfügung, die für die Korrektheit der intuitiven Überzeugungen entscheidend sind. Durch das auf der Ebene des Typ 1-Denkens stattfindende Ablesen des mentalen Modells ergibt sich eine Gehaltswiederholung, womit (AW) erfüllt ist. Dass sich diese Wiederholung auf der subpersonalen Ebene ereignet, ist hierbei kein Grund dafür, sie als irrelevant zu betrachten, da im Modell intuitiver Rechtfertigung andere Elemente hinzutreten, welche die Verfügbarkeit des Grundes sicherstellen.

Die Wiederholung der Gehalte im Prozess des Ablesens lässt sich durch eine Analogie veranschaulichen: Eine Ermittlerin macht eine Fotografie von einem 
Tatort, auf der bereits wichtige Bezüge von Elementen zueinander ersichtlich sind, die ihr im Moment des Fotografierens noch nicht auffallen. Durch das spätere Betrachten des Fotos können ihr diese Bezüge bewusstwerden. Auch im Modell intuitiver Rechtfertigung wiederholen sich somit Elemente, nämlich die nichtbegrifflichen Gehalte des mentalen Modells durch den Vorgang des Ablesens. Somit kann (AW) akzeptiert werden, ohne auf begrifflichen Gehalten bestehen zu müssen.

Eine direkte Reaktion der Vertreter der inferentiellen Isolation nichtbegrifflicher Gehalte auf diese Ausführungen bestünde vermutlich darin zu insistieren, dass eine solche Wiederholung eine Wiederholung für das Subjekt sein muss und daher nur begrifflich zu denken ist. Als Replik darauf kann auf die mehrfach im Modell intuitiver Rechtfertigung einfließende Bekanntschaftsrelation verwiesen werden. Durch diese Relation wird auch die im Modell angelegte Wiederholung zu einem mit dem Subjekt in Verbindung stehenden Element, ohne dass dieses begrifflich vorliegen muss.

Ein weiteres Bedenken gegen die hier vorgelegte Erklärung bestünde wohl darin, dass dem für Rechtfertigungsbeziehungen notwendigen Holismus (bei den Begriffen erfüllt durch deren Einbettung in ein Begriffsnetz) allein durch (AW) noch nicht genügend Rechnung getragen wird. Nach dieser Auffassung ist der inferentielle Bezug zu anderen Gehalten das Merkmal der Begrifflichkeit schlechthin. Das Bedenken ist nun, dass ein solcher Holismus in Bezug auf nichtbegriffliche Gehalte nicht gegeben ist. Dies wäre vertieft zu untersuchen, jedoch lässt sich bereits festhalten: Dass nichtbegriffliche Gehalte nicht in gleicher Weise holistisch zu denken sind, ist keineswegs sicher. Vielmehr scheint es - unterstützt durch die Theorie der mentalen Modelle - äußerst plausibel, auch in jedem Fall einer Repräsentation mit nichtbegrifflichem Gehalt zu sagen, dass diese erst durch andere Repräsentationen ermöglicht wird. Nach dieser Hypothese kann ein Subjekt nicht nur unmöglich eine einzige Überzeugung haben, sondern es kann auch unmöglich nur einen einzigen Gehalt repräsentieren, da jeder Gehalt in ein mentales Modell eingebettet ist, was ihn zu einem wesentlichen Teil charakterisiert. So ist beispielsweise die Windrichtung im Feuerwehrbeispiel erst dann - auch nichtbegrifflich repräsentierbar, wenn sie zu räumlichen Repräsentationen (oben, unten,...) in Bezug steht. Da dies eine plausible Charakterisierung der Situation ist, reicht der Verweis auf den Holismus im Bereich der Überzeugungen nicht aus, um die inferentielle Isolation nichtbegrifflicher Gehalte zu belegen.

Aufgrund dieser Überlegungen scheint die These der inferentiellen Isolation nichtbegrifflicher Gehalte, sofern sie nicht auf eine bloße Wiederholung von Vorurteilen zur Deckungsgleichheit zwischen Begrifflichkeit 
und Begründung hinauslaufen soll, argumentativ nicht ausreichend gestützt zu sein. Zunächst ist die Wiederholung von Elementen auch bei der Rechtfertigung intuitiver Überzeugungen gegeben, wie durch die Theorie der mentalen Modelle erwiesen wird. Das Modell intuitiver Rechtfertigung erfüllt somit (AW). Auch weitreichendere Forderungen nach einem Holismus von Gehalten lassen sich nicht ausschließlich als durch begriffliche Gehalte erfüllt sehen. Daher bleibt die im Modell intuitiver Rechtfertigung postulierte Gründe-Beziehung zwischen nichtpropositionaler Intuition und intuitiver Überzeugung unangefochten. 